\title{
Bayesian Model-Based Clustering Procedures
}

\author{
John W. LAU and Peter J. GREeN
}

\begin{abstract}
This article establishes a general formulation for Bayesian model-based clustering, in which subset labels are exchangeable, and items are also exchangeable, possibly up to covariate effects. The notational framework is rich enough to encompass a variety of existing procedures, including some recently discussed methods involving stochastic search or hierarchical clustering, but more importantly allows the formulation of clustering procedures that are optimal with respect to a specified loss function. Our focus is on loss functions based on pairwise coincidences, that is, whether pairs of items are clustered into the same subset or not.

Optimization of the posterior expected loss function can be formulated as a binary integer programming problem, which can be readily solved by standard software when clustering a modest number of items, but quickly becomes impractical as problem scale increases. To combat this, a new heuristic item-swapping algorithm is introduced. This performs well in our numerical experiments, on both simulated and real data examples. The article includes a comparison of the statistical performance of the (approximate) optimal clustering with earlier methods that are model-based but ad hoc in their detailed definition.
\end{abstract}

Key Words: Dirichlet process; Hierarchical clustering; Loss functions; Stochastic search.

\section{INTRODUCTION}

Clustering methods aim to separate a heterogeneous collection of items into homogeneous subsets and are an important tool in scientific investigations in many different domains. There is a particular focus of activity in recent years, as biologists have become interested in clustering high-dimensional data generated by modern high-throughput assay techniques: for example, in order to cluster genes on the basis of gene expression measurements from microarrays. With the rapid growth in computer power, Bayesian statisticians have become able to implement principled computer-intensive inferential methods for clustering based, for example, on mixture models (see Fraley and Raftery 2002; Medvedovic and Sivaganesan 2002; Medvedovic et al. 2004; Yeung et al. 2001).

John W. Lau is Lecturer, School of Statistics and Actuarial Science, University of the Witwatersrand, Private Bag 3, 2050 WITS, Johannesburg, South Africa (E-mail: john.w.lau@googlemail.com). Peter J. Green is Professor of Statistics, Department of Mathematics, University of Bristol, Bristol, BS8 1TW, United Kingdom (E-mail: P.J.Green@bristol.ac.uk).

\footnotetext{
(C) 2007 American Statistical Association, Institute of Mathematical Statistics, and Interface Foundation of North America

Journal of Computational and Graphical Statistics, Volume 16, Number 3, Pages 526-558

DOI: $10.1198 / 106186007 X 238855$
} 
In a Bayesian formulation of a clustering procedure, the partition of items into subsets becomes a parameter of a probability model for the data, subject to prior assumptions, and inference about the clustering derives from properties of the posterior distribution. There are the usual opportunities for simultaneous inference about the partition and other parameters, characterizing the cluster locations and spreads, for example, but our principal focus in this article is the partition itself. In contrast to most other unknowns in a Bayesian model, where the posterior mean provides an often adequate default choice of point estimator, there is no simple natural choice for an estimator of a partition. Unlike the case of classical approaches, such as hierarchical clustering and $K$-means clustering procedures, Bayesian alternatives seem not to be fully developed.

Medvedovic and Sivaganesan (2002) and Medvedovic et al. (2004) generated a sample of partitions based on the Dirichlet process kernel mixture model. They used this to estimate a pairwise similarity matrix and employed classical procedures (see Gordon 1999) to define a "best" partition. Somewhat similarly, Dubey et al. (2004) used a Dirichlet process mixture model on 10-dimensional data derived from an analysis of protein sequences; posterior probabilities of clustering are then input to a hierarchical clustering algorithm. Again starting from a Dirichlet process kernel mixture model, but with some clever choices to expedite the computations, Dahl (2006) selected a partition from an MCMC algorithm that minimizes the sum of squared deviations from the pairwise probability matrix (of probabilities that a pair lie in the same cluster). The procedure searches for a partition subject by minimizing this objective function. Recently, Ray and Mallick (2006) developed a semiparametric wavelet model with parameters distributed according to a Dirichlet process. They took a marginal likelihood approach, proposed by Basu and Chib (2003), to choose a partition of the data. Other relevant work, by Heard et al. (2006) and by Heller and Gharamani (2005), will be discussed in Section 3.

Choosing a partition can be considered as a model choice problem, for which the usual Bayesian tools are marginal likelihoods and Bayes factors. Both of these essentially involve considering posterior probabilities under a uniform prior. This might be questioned on three grounds: the arbitrariness of taking a uniform prior, the fact that peaks of posterior probability in a high-dimensional problem can be isolated and unrepresentative, and the problem that posterior probabilities of individual partitions are difficult to compute reliably from Monte Carlo samples.

A loss function approach avoids these criticisms, and delivers an objective procedure. Binder (1978, 1981) discussed loss functions for Bayesian clustering, although many of his choices are not appropriate for our setting, as we wish to respect exchangeability a priori in the labeling of clusters and of items. We therefore concentrate on loss functions defined on pairwise coincidences. We penalize pairs of items that are assigned to different clusters when they should be in the same one, and vice versa; the total loss is obtained by summing over all pairs. The resulting posterior expected loss is a simple linear function of posterior marginal coincidence probabilities (i.e., for each pair the posterior probability they are clustered together), which are readily estimated from a Markov chain Monte Carlo (MCMC) simulation from the posterior. See also Rue (1995) for an important discussion of loss functions for high-dimensional Bayesian models with inference computed by MCMC, 
although his detailed proposals do not seem relevant to our context.

Section 2 introduces the Bayesian partition model under a general random measure prior. The prior and likelihood parts of the partition model are discussed separately. Section 3 discusses two existing approaches to computing a point estimate of a partition-a naive stochastic search approach and a hierarchical Bayesian procedure. We also make numerical comparisons.

We show in Section 4 that optimization of this expected loss is a binary integer programming problem. Standard techniques are far too slow for practical use for large numbers $n$ of items (say of the order of 1,000), since the size of the integer program grows rapidly (the numbers of constraints as $n^{3}$, and of variables as $n^{2}$ ), and so approximations are required.

Our methods are illustrated on both simulated and real datasets. The simulated data are generated from bivariate normal mixtures with from one to four components. The real data used are the galaxy data from Roeder (1990), and gene expression data from a leukemia study reported by Golub et al. (1999).

\section{BAYESIAN CLUSTERING MODELS}

A partition $\mathbf{p}$ separates $n$ items with observed responses $\mathbf{y}=\left\{\mathbf{y}_{1}, \ldots, \mathbf{y}_{n}\right\}$ into subsets. Such a partition is represented as $\left\{C_{1}, \ldots, C_{n(\mathbf{p})}\right\}$ where $C_{j}$ denotes the $j$ th subset or cluster, for $j=1, \ldots, n(\mathbf{p})$. Each $C_{j}$ contains indices of the items in that cluster, and the positive integer $n(\mathbf{p})$ denotes the number of clusters, which depends of course on the partition. There is a positive number $e_{j}$ of items in each cluster $j$. Given the partition $\mathbf{p}$ of items, a model for the data is defined as

$$
\pi(\mathbf{y} \mid \mathbf{p})=\prod_{j=1}^{n(\mathbf{p})} m\left(\left\{\mathbf{y}_{i}, i \in C_{j}\right\}\right)=\prod_{j=1}^{n(\mathbf{p})} m\left(\mathbf{y}_{C_{j}}\right)
$$

say, where $m\left(\mathbf{y}_{C_{j}}\right)$ is the joint distribution of the responses for items in cluster $C_{j}$. We preserve exchangeability across cluster labels and item indexing by requiring this function not to depend on $j$ and, typically, to be an exchangeable function of its arguments $\left\{\mathbf{y}_{i}, i \in\right.$ $C_{j}$ \}. Both these kinds of exchangeability follow usual practice in clustering problems. Naturally, the demands of specific contexts may demand variations in these assumptions; for example, in separate work we are adapting our models and criteria to gene expression profile clustering, allowing a "background" cluster not exchangeable with the others.

Since we also wish to perform clustering in the presence of covariate information $\left\{\mathbf{x}_{i}, i=1,2, \ldots, n\right\}$ on the items, we allow $m\left(\mathbf{y}_{C_{j}}\right)$ to depend tacitly on $\left\{\mathbf{x}_{i}, i \in C_{j}\right\}$, and exchangeability over items then relates to permuting $\left(\mathbf{y}_{i}, \mathbf{x}_{i}\right)$ pairs jointly. Typically, we suppose

$$
m\left(\mathbf{y}_{C_{j}}\right)=\int_{\mathcal{U}} \prod_{i \in C_{j}} k\left(\mathbf{y}_{i} \mid u_{j}\right) G_{0}\left(d u_{j}\right) \quad \text { or } \quad \int_{\mathcal{U}} \prod_{i \in C_{j}} k\left(\mathbf{y}_{i} \mid u_{j} ; \mathbf{x}_{i}\right) G_{0}\left(d u_{j}\right)
$$

and $k\left(\mathbf{y}_{i} \mid u_{j}\right)$ is a density for $\mathbf{y}_{i}$ s with parameters/latent variables $u_{j}$ and $G_{0}$ is a distribution function of $u_{j}$ on the space $\mathcal{U}$. 
Equation (2.2), which guarantees the required exchangeability properties, may be viewed either as a formal representation (de Finetti 1930, 1974; Hewitt and Savage 1955; Diaconis and Freedman 1984, 1987), or as an ingredient in the specification of a Bayesian hierarchical model, in which clusters are assigned characterising parameters $u_{j}$, iid from distribution $G_{0}$, and item responses within clusters drawn independently from distribution $k\left(\mathbf{y}_{i} \mid u_{j}\right)$ or $k\left(\mathbf{y}_{i} \mid u_{j} ; \mathbf{x}_{i}\right)$.

In the full Bayesian formulation, a prior probability would be assigned to each partition $\mathbf{p}$, leading to a posterior of the form

$$
\pi(\mathbf{p} \mid \mathbf{y}) \propto \phi(\mathbf{p})=\pi(\mathbf{p}) \prod_{j=1}^{n(\mathbf{p})} m\left(\mathbf{y}_{C_{j}}\right) .
$$

[References include Hartigan (1990), Barry and Hartigan (1992), and Quintana and Iglesias (2003), who regarded this as a product partition model $(\mathcal{P P} \mathcal{M})$. See also Brunner and Lo (1999) and Lo (2005)]

Of key importance to practical inference in this model setting is the question whether $m\left(\mathbf{y}_{C_{j}}\right)$ is explicitly known or not. When it is, MCMC procedures are greatly simplified, since cluster-specific parameters $u_{j}$ can be integrated out, and the posterior distribution of $\mathbf{p}$ alone (or possibly $\mathbf{p}$ together with a few hyperparameters) can be the target of an MCMC sampler. For the remainder of this article, we will suppose that $m\left(\mathbf{y}_{C_{j}}\right)$ is explicitly available: in the context of (2.2) this amounts to assuming that $G_{0}$ is conjugate for the density $k\left(\mathbf{y}_{i} \mid \cdot\right)$.

\subsection{The Prior Part of the Clustering Model}

In the absence of real prior information about the items, we will assign positive prior probability to every possible partition. In the interests of computational convenience, we might be attracted to prior models for which posterior simulation methods are fully developed, and this leads us to models based on random probability measures. The Ferguson (1973) Dirichlet process (see also Antoniak 1974) is a popular choice, suggested by Lo (1984). Ishwaran and James (2001, 2003a, 2003b) employed stick breaking random probability measures, including the Kingman-Pitman-Yor Poisson-Dirichlet process, as a prior for the kernel mixture model. Another familiar example is the finite mixture model with $\kappa$ components, and a symmetric Dirichlet distribution with parameters $(\delta, \delta, \ldots, \delta)$ on the component weights; this yields an explicit prior on the partition when the weights are integrated out. For these models, the corresponding $\pi(\mathbf{p})$ s are

$\begin{array}{lll}\text { Random Probability Measures } & \text { Parameters } & \pi(\mathbf{p}) \propto \\ \text { Dirichlet process } & \theta>0 & \theta^{n(\mathbf{p})} \prod_{j=1}^{n(\mathbf{p})} \Gamma\left(e_{j}\right) \\ \text { Finite mixture model, Dirichlet weights } & \kappa, \delta>0 & \frac{\kappa !}{\kappa-n(\mathbf{p})) !} \prod_{j=1}^{n(\mathbf{p})} \frac{\Gamma\left(\delta+e_{j}\right)}{\Gamma(\delta)} \\ \text { Poisson-Dirichlet process } & \theta>-\alpha, 0 \leq \alpha<1 & \prod_{j=1}^{n(\mathbf{p})}\left[(\theta+\alpha(j-1)) \frac{\Gamma\left(e_{j}-\alpha\right)}{\Gamma(1-\alpha)}\right] .\end{array}$


James (2002, 2005) and Lijoi et al. (2005) considered more general construction of kernel mixtures based on certain classes of random probability measures. For more random probability measures, we refer to Kingman (1975, 1993), Pitman (1995, 1996, 2003), as well as Pitman and Yor (1997).

In fact, however, in the conjugate setting, posterior simulation is equally straightforward for much wider classes of prior, and all that is needed for implementation is the availability of the ratio of prior probabilities $\pi\left(\mathbf{p}^{\prime}\right) / \pi(\mathbf{p})$ when $\mathbf{p}^{\prime}$ is obtained from $\mathbf{p}$ by reassigning a single item. Thus, given prior information, we are free to assign prior probabilities $\pi$ (p) to suit the situation; the procedures discussed in this article will still work for most choices.

\subsection{The Data Part of the Clustering Model}

We discuss two kinds of model (2.2). One of them follows standard Bayesian modelling lines, taking the density $k$ of (2.2) to be normal with unknown mean and unknown variance/precision, and $G_{0}$ as the (Normal-Gamma) parameter prior. Then $m\left(\mathbf{y}_{C_{j}}\right)$ will be a $t$-density. For the other kind of model, we directly assign $m\left(\mathbf{y}_{C_{j}}\right)$ to be an exchangeable density of $\mathbf{y}_{C_{j}}$. This can be a convenient way to proceed, and also allows us to make connections with the classical theory of clustering.

Normal-Gamma set up. Motivated by application to clustering gene expression profiles, we discuss a regression-type model that expresses a vector response in terms of a linear combination of known covariates. We take $\mathbf{y}_{i}$ as a vector, written as $\mathbf{y}_{i}=\left[y_{i 1} \ldots y_{i}\right]^{\prime}$ for $i=1, \ldots, n$. Given the covariates $\mathbf{x}_{s}=\left[x_{s 1} \ldots x_{S K}\right]^{\prime}$ and that $i$ lies in the cluster $j$, the parameters/latent variables are $\boldsymbol{\beta}_{j}=\left[\beta_{j 1} \ldots \beta_{j K}\right]^{\prime}$ and $\tau_{j}$. The regression part of the model can be written as

$$
\mathbf{y}_{i}=\left[\begin{array}{c}
y_{i 1} \\
\vdots \\
y_{i S}
\end{array}\right]=\sum_{k=1}^{K} \beta_{j k}\left[\begin{array}{c}
x_{1 k} \\
\vdots \\
x_{S k}
\end{array}\right]+\left[\begin{array}{c}
\epsilon_{i 1} \\
\vdots \\
\epsilon_{i S}
\end{array}\right]=\left[\mathbf{x}_{1} \ldots \mathbf{x}_{S}\right]^{\prime} \boldsymbol{\beta}_{j}+\boldsymbol{\epsilon}_{i},
$$

where $\boldsymbol{\epsilon}_{i} \sim N\left(\mathbf{0}_{S \times 1}, \tau_{j}^{-1} \mathbf{I}_{S \times S}\right)$, where $\mathbf{0}_{S \times 1}$ is the $S$-dimensional zero vector and $\mathbf{I}_{S \times S}$ is the $S \times S$ identity matrix. The covariates $\left[\mathbf{x}_{1} \ldots \mathbf{x}_{S}\right]^{\prime}$ are fixed and observed, and may as usual include dummy covariates for factor levels. It could be an identity matrix (when $K=S$ ). From (2.4), $\mathbf{y}_{i}$ follows a multivariate Normal density with mean $\boldsymbol{\beta}_{j}$ and variance $\tau_{j}^{-1} \mathbf{I}_{S \times S}$. Writing $u_{j}=\left(\boldsymbol{\beta}_{j}, \tau_{j}\right)$, the kernel is represented as $k\left(\mathbf{y}_{i} \mid \boldsymbol{\beta}_{j}, \tau_{j}\right)$, a multivariate normal density, $N\left(\left[\mathbf{x}_{1} \ldots \mathbf{x}_{S}\right]^{\prime} \boldsymbol{\beta}_{j}, \tau_{j}^{-1} \mathbf{I}_{S \times S}\right)$. To complete the Normal set up, given $\tau_{j}, \boldsymbol{\beta}_{j}$ follows the $K$-dimensional multivariate normal with mean $\mathbf{m}_{0}$ and variance $\left(\tau_{j} \mathbf{t}_{0}\right)^{-1}$, and $\tau_{j}$ follows the univariate Gamma with shape $a_{0}$ and scale $b_{0}$. We denote the joint distribution $G_{0}\left(d \boldsymbol{\beta}_{j}, d \tau_{j}\right)$ as a joint Gamma-Normal distribution, Gamma-Normal $\left(a_{0}, b_{0}, \mathbf{m}_{0}, \mathbf{t}_{0}\right.$. 
Based on the set up, we have

$$
\begin{aligned}
m\left(\mathbf{y}_{C_{j}}\right)= & \frac{\Gamma\left(\frac{2 a_{0}+e_{j} S}{2}\right)}{\Gamma\left(\frac{2 a_{0}}{2}\right)} \frac{\left|\frac{b_{0}}{a_{0}}\left(\mathbf{X}_{C_{j}} \mathbf{t}_{0}^{-1} \mathbf{X}_{C_{j}}^{\prime}+\mathbf{I}_{e_{j} S \times e_{j} S}\right)\right|^{-1 / 2}}{\left(2 a_{0} \pi\right)^{e_{j} S / 2}} \\
& \times\left(1+\frac{1}{2 a_{0}}\left(\mathbf{y}_{C_{j}}-\mathbf{X}_{C_{j}} \mathbf{m}_{0}\right)^{\prime}\left(\frac{b_{0}}{a_{0}}\left(\mathbf{X}_{C_{j}} \mathbf{t}_{0}^{-1} \mathbf{X}_{C_{j}}^{\prime}+\mathbf{I}_{e_{j} S \times e_{j} S}\right)\right)^{-1}\right. \\
& \left.\times\left(\mathbf{y}_{C_{j}}-\mathbf{X}_{C_{j}} \mathbf{m}_{0}\right)\right)^{-\left(2 a_{0}+e_{j} S\right) / 2}
\end{aligned}
$$

where $\mathbf{y}_{C_{j}}=\left[\mathbf{y}_{i_{1}}^{\prime} \ldots \mathbf{y}_{i_{e_{j}}}^{\prime}\right]^{\prime}$ and $\mathbf{X}_{C_{j}}=\left[\left[\mathbf{x}_{1} \ldots \mathbf{x}_{S}\right] \ldots\left[\mathbf{x}_{1} \ldots \mathbf{x}_{S}\right]\right]^{\prime}$ for $C_{j}=\left\{i_{1}, \ldots, i_{e_{j}}\right\}$. Note that $\mathbf{y}_{C_{j}}$ is a $e_{j} S$ vector and $\mathbf{X}_{C_{j}}$ is a $e_{j} S \times K$ matrix. Moreover, $m\left(\mathbf{y}_{C_{j}}\right)$ is a multivariate $t$ density with mean $\mathbf{X}_{C_{j}} \mathbf{m}_{0}$, scale $\frac{b_{0}}{a_{0}}\left(\mathbf{X}_{C_{j}} \mathbf{t}_{0}^{-1} \mathbf{X}_{C_{j}}^{\prime}+\mathbf{I}_{e_{j} S \times e_{j} S}\right)$ and degrees of freedom $2 a_{0}$.

Directly assigned marginals approach. Alternatively, we can directly assign $m\left(\mathbf{y}_{C_{j}}\right)$ to be some exchangeable (symmetric) function, that is nonnegative and integrable. One interesting construction is

$$
m\left(\mathbf{y}_{C_{j}}\right)=e^{-\psi\left(\mathbf{y}_{C_{j}}\right)},
$$

where the function $\psi$ is nonnegative and exchangeable. A popular choice of function $\psi$ is

$$
\psi\left(\mathbf{y}_{C_{j}}\right)=w_{j} \times \sum_{i \in C_{j}}\left(\mathbf{y}_{i}-\overline{\mathbf{y}}_{C_{j}}\right)^{\prime}\left(\mathbf{y}_{i}-\overline{\mathbf{y}}_{C_{j}}\right),
$$

where

$$
\overline{\mathbf{y}}_{C_{j}}=\frac{1}{e_{j}} \sum_{i \in C_{j}} \mathbf{y}_{i}
$$

Usually, we would like to choose $w_{j}=1$ and the $\psi$ is related to the sum of squared errors within each cluster. This gives a stochastic version of the Ward (1963) clustering model. Moreover, $w_{j}=e_{j}$ gives us an interesting case, that is

$$
\psi\left(\mathbf{y}_{C_{j}}\right)=e_{j} \times \sum_{i \in C_{j}}\left(\mathbf{y}_{i}-\overline{\mathbf{y}}_{C_{j}}\right)^{\prime}\left(\mathbf{y}_{i}-\overline{\mathbf{y}}_{C_{j}}\right)=\sum_{i_{1}<i_{2}, i_{1}, i_{2} \in C_{j}} d_{i_{1}, i_{2}},
$$

where

$$
d_{i_{1}, i_{2}}=\left(\mathbf{y}_{i_{1}}-\mathbf{y}_{i_{2}}\right)^{\prime}\left(\mathbf{y}_{i_{1}}-\mathbf{y}_{i_{2}}\right) .
$$

These models focus on the sum of the pairwise distances of all pairs in each cluster. See also Banfield and Raftery (1993) for some existing models. For an analogue of (2.5), we could take

$$
\psi\left(\mathbf{y}_{C_{j}}\right)=\sum_{i \in C_{j}}\left(\mathbf{y}_{i}-\left[\mathbf{x}_{1} \ldots \mathbf{x}_{S}\right]^{\prime} \hat{\boldsymbol{\beta}}_{C_{j}}\right)^{\prime}\left(\mathbf{y}_{i}-\left[\mathbf{x}_{1} \ldots \mathbf{x}_{S}\right]^{\prime} \hat{\boldsymbol{\beta}}_{C_{j}}\right),
$$


where

$$
\hat{\boldsymbol{\beta}}_{C_{j}}=\left(\mathbf{X}_{C_{j}}^{\prime} \mathbf{X}_{C_{j}}\right)^{-1} \mathbf{X}_{C_{j}}^{\prime} \mathbf{y}_{C_{j}}
$$

This is an extension from the random sample case (2.7) to the regression case. It is also minus twice the exponent of a normal density which is a limiting case of (2.5), in which we take $b_{0}=a_{0}, \mathbf{t}_{0}=\mathbf{0}, \mathbf{m}_{0}=\mathbf{0}$, and $2 a_{0} \rightarrow \infty$. Note that (2.10) is valid for any size of cluster if and only if $S \geq K$; otherwise estimators other than least squares could be used.

\section{CLUSTERING PROCEDURES}

MCMC procedures deliver an approximation to the posterior of the partition $\mathbf{p}$, but for many purposes a single point estimate $\hat{\mathbf{p}}$ will be sought. In the following section, we consider optimal point estimates based on loss functions, but using the posterior alone, perhaps the only reasonable estimate is the posterior mode, maximizing (2.3). Two main kinds of approach have been considered recently, and will be discussed in the section.

First, stochastic search methods simply run a MCMC sampler in equilibrium for the posterior distribution, and record the $\mathbf{p}$ with highest posterior probability encountered during the run. For example, Brunner and Lo (1999) did this for a Dirichlet process model, using the usual Gibbs sampler for the partition allocation variables (the "weighted Chinese restaurant process" procedure). Recently, Nobile and Fearnside (2005) proposed an allocation sampler which seems to be an efficient alternative sampler for such models, although their setting is the finite mixture model with Multinomial-Dirichlet prior. Other than the sampler used here, stochastic search may be performed using other MCMC samplers over the posterior distribution. Examples include the samplers proposed by West et al. (1994) and Richardson and Green (1997) for mixture models. The chief weakness of such stochastic search schemes is that, since the number of possible partitions (given by the Bell number) is huge, it is impossible to visit all possible partitions to identify $\hat{\mathbf{p}}$. Moreover, it seems intractable to produce proper summary statistics, say frequency counts, of the visited partitions as the set of visited partitions may be very irregular. Yet, we may encounter a partition close to $\hat{\mathbf{p}}$ fortuitously. A more disciplined but computationally intensive MCMC-based approach to optimization is simulated annealing (Kirkpatrick et al. 1983), but we do not pursue this here.

Heard et al. (2006) and Heller and Ghahramani (2005) have both avoided MCMC sampling in approximating the posterior mode in a Bayesian clustering model. Bayesian hierarchical clustering is a deterministic hierarchical agglomerative model-based procedure. Here we follow in detail Heard et al. (2006) who used finite mixture models over a Multinomial-Dirichlet prior. The idea is simple: two clusters are combined iteratively to maximize the posterior probability (2.3). A sequence of partitions is built up from $n$ singleton clusters initially, terminating in a single cluster. As the number of clusters $n$ (p) decreases, the values of the posterior probability (2.3) first rises and then falls again; the best partition over this sequence can then be discovered. 


\subsection{Stochastic Search by Posterior Sampling of Partitions}

We briefly discuss the Gibbs sampler for allocation variables, also known as the weighted Chinese restaurant process procedure. The objective is to sample $\mathbf{p}$ from the stationary distribution $\pi$ (p|y) (see Escobar and West 1995, 1998; Ishwaran and James 2001, 2003a, 2003b; Lo et al. 1996; MacEachern 1994; MacEachern and Müller 1998, 2000; Neal 2000; West et al. 1994; and so on). Here we simply introduce the procedures. Given a partition $\mathbf{p}$, one item, say $k$, is removed, leaving a partition of $n-1$ items which we denote as $\mathbf{p}_{-k}=\left\{C_{1,-k}, \ldots, C_{n\left(\mathbf{p}_{-k}\right),-k}\right\}$. The size of each cluster is $e_{j,-k}$ for $j=1, \ldots, n\left(\mathbf{p}_{-k}\right)$ and the number of clusters is $n\left(\mathbf{p}_{-k}\right)$. The item will be assigned to a new cluster with probability proportional to

$$
m\left(\mathbf{y}_{\{k\}}\right) \times \begin{cases}\theta & \text { for the Dirichlet process } \\ \left(\kappa-n\left(\mathbf{p}_{-k}\right)\right) \delta & \text { for the finite mixture model } \\ \theta+\alpha n\left(\mathbf{p}_{-k}\right) & \text { for the Poisson-Dirichlet process } \\ 1 & \text { for the Uniform Prior }\end{cases}
$$

and the item will be assigned to the cluster $j$ with probability proportional to

$$
\frac{m\left(\mathbf{y}_{\left.\{k\} \cup C_{j,-k}\right)}\right)}{m\left(\mathbf{y}_{C_{j,-k}}\right)} \times \begin{cases}e_{j,-k} & \text { for the Dirichlet process } \\ e_{j,-k}+\delta & \text { for the finite mixture model } \\ e_{j,-k}-\alpha & \text { for the Poisson-Dirichlet process } \\ 1 & \text { for the Uniform Prior }\end{cases}
$$

for $j=1, \ldots, n\left(\mathbf{p}_{-k}\right)$. In general, the sampler can be described as follows. The item will be assigned to a new cluster with probability proportional to

$$
m\left(\mathbf{y}_{\{k\}}\right) \times \frac{\pi\left(C_{1,-k}, \ldots, C_{n\left(\mathbf{p}_{-k}\right),-k},\{k\}\right)}{\pi\left(C_{1,-k}, \ldots, C_{n\left(\mathbf{p}_{-k}\right),-k}\right)}
$$

and the item will be assigned to the cluster $j$ with probability proportional to

$$
\frac{m\left(\mathbf{y}_{\{k\} \cup C_{j,-k}}\right)}{m\left(\mathbf{y}_{C_{j,-k}}\right)} \times \frac{\pi\left(C_{1,-k}, \ldots, C_{j,-k} \cup\{k\}, \ldots, C_{n\left(\mathbf{p}_{-k}\right),-k}\right)}{\pi\left(C_{1,-k}, \ldots, C_{j,-k}, \ldots, C_{n\left(\mathbf{p}_{-k}\right),-k}\right)}
$$

for $j=1, \ldots, n\left(\mathbf{p}_{-k}\right)$.

As an alternative to the weighted Chinese restaurant process procedure, Nobile and Fearnside (2005) proposed a series of procedures to sample from a partition model generated by a finite mixture models with a Multinomial-Dirichlet prior in order to enhance the mixing of the sampler. Inspired by the reversible jump sampling idea, it is an efficient Metropolis-Hastings sampler for reallocating items among partitions. Here we mention two related moves. The Absorption/Merge Move proposes combining two randomly chosen clusters into one; the Ejection/Split Move is the reverse move. If the two clusters concerned are $C_{j_{1}}$ and $C_{j_{2}}$, the acceptance probabilities for these moves are $\min \{1, R\}$ and $\min \left\{1, R^{-1}\right\}$, where in general,

$$
R=\frac{m\left(\mathbf{y}_{C_{j_{1}} \cup C_{j_{2}}}\right)}{m\left(\mathbf{y}_{C_{j_{1}}}\right) m\left(\mathbf{y}_{C_{j_{2}}}\right)} \frac{\pi\left(C_{1}, \ldots, C_{j_{1}} \cup C_{j_{2}}, \ldots, C_{n(\mathbf{p})}\right)}{\pi\left(C_{1}, \ldots, C_{n(\mathbf{p})}\right)} .
$$

Other moves are available by systematically transferring items between partition subsets. 


\subsection{Bayesian Hierarchical Clustering Procedures}

We will use our model (2.3) to present the idea of the deterministic hierarchical agglomerative model-based clustering procedure of Heard et al. (2006), which can generally be computed very cheaply compared to methods requiring Monte Carlo sampling over partitions. There are some interesting features that appear only in partition models generated by the class of random probability measures, for example, the Dirichlet process and Poisson-Dirichlet process. We initiate the procedure with all singleton clusters, and step-by-step, combine pairs of clusters until all items are clustered together.

The iterative step is at each stage to combine the two clusters $C_{j_{1}}$ and $C_{j_{2}}$ that maximize

$$
\frac{m\left(\mathbf{y}_{C_{j_{1}} \cup C_{j_{2}}}\right)}{m\left(\mathbf{y}_{C_{j_{1}}}\right) m\left(\mathbf{y}_{C_{j_{2}}}\right)} \times \eta\left(e_{j_{1}}, e_{j_{2}}\right)
$$

where

$$
\eta\left(e_{j_{1}}, e_{j_{2}}\right)= \begin{cases}\frac{\Gamma\left(e_{j_{1}}+e_{j_{2}}\right)}{\Gamma\left(e_{j_{1}}\right) \Gamma\left(e_{j_{2}}\right)} & \text { for the Dirichlet process } \\ \frac{\Gamma\left(e_{j_{1}}+e_{j_{2}}+\delta\right)}{\Gamma\left(e_{j_{1}}+\delta\right) \Gamma\left(e_{j_{2}}+\delta\right)} & \text { for the finite mixture model } \\ \frac{\Gamma\left(e_{j_{1}}+e_{j_{2}}-\alpha\right)}{\Gamma\left(e_{j_{1}}-\alpha\right) \Gamma\left(e_{j_{2}}-\alpha\right)} & \text { for the Poisson-Dirichlet process } \\ 1 & \text { for the Uniform Prior }\end{cases}
$$

This choice of clusters to combine maximizes the posterior quantity (2.3) among all partitions that can be obtained from the present one by merging a pair of clusters. Suppose we move $\mathbf{p}=\left\{C_{1}, \ldots, C_{n(\mathbf{p})}\right\}$ to $\mathbf{p}^{*}=\left\{C_{1}^{*}, \ldots, C_{n\left(\mathbf{p}^{*}\right)}^{*}\right\}$ by combining the clusters $C_{j_{1}}$ and $C_{j_{2}}$, where $e_{j}$ for $j=1, \ldots, n(\mathbf{p})$ and $e_{j}^{*}$ for $j=1, \ldots, n\left(\mathbf{p}^{*}\right)$ are the size of clusters, respectively. Then, from (2.3), we find:

$$
\phi\left(\mathbf{p}^{*}\right)=\phi(\mathbf{p}) \times\left(\frac{1}{\xi} \eta\left(e_{j_{1}}, e_{j_{2}}\right) \frac{m\left(\mathbf{y}_{C_{j_{1}} \cup C_{j_{2}}}\right)}{m\left(\mathbf{y}_{C_{j_{1}}}\right) m\left(\mathbf{y}_{C_{j_{2}}}\right)}\right),
$$

where

$$
\xi= \begin{cases}\frac{\theta-(n(\mathbf{p})-1)) \delta}{\Gamma(\delta+1)} & \text { for the Dirichlet process } \\ \frac{\theta+\alpha(n(\mathbf{p})-1)}{\Gamma(1-\alpha)} & \text { for the Poinite mixture model } \\ 1 & \text { for the Uniform Prior. }\end{cases}
$$

In practice, Heard et al. (2006) constructed a sequence of partitions from the all-singletons partition to a single cluster partition, and then determine the best partition by comparisons though (2.3). Note that the maximizing process does not depend on the quantity $\xi$. 
We now consider particular cases of this procedure for specific directly assigned choices of $m\left(\mathbf{y}_{C_{j}}\right)$. If we choose $m\left(\mathbf{y}_{C_{j}}\right)$ in (2.2), to be given by (2.6) and (2.7), and tailormake $\pi(\mathbf{p}) \propto \theta^{n(\mathbf{p})}$ for an assumed value of $\theta$, the procedure works as follows. Iteratively, we combine two clusters $C_{j_{1}}$ and $C_{j_{2}}$ if

$$
\begin{aligned}
& \frac{e_{j_{1}} e_{j_{2}}}{e_{j_{1}}+e_{j_{2}}}\left(\overline{\mathbf{y}}_{C_{j_{1}}}-\overline{\mathbf{y}}_{C_{j_{2}}}\right)^{\prime}\left(\overline{\mathbf{y}}_{C_{j_{1}}}-\overline{\mathbf{y}}_{C_{j_{2}}}\right) \\
& \quad \leq \min _{j_{2}, j_{1} \in\{1, \ldots, n(\mathbf{p})\}, j_{1} \neq j_{2}}\left\{\frac{e_{j_{1}} e_{j_{2}}}{e_{j_{1}}+e_{j_{2}}}\left(\overline{\mathbf{y}}_{C_{j_{1}}}-\overline{\mathbf{y}}_{C_{j_{2}}}\right)^{\prime}\left(\overline{\mathbf{y}}_{C_{j_{1}}}-\overline{\mathbf{y}}_{C_{j_{2}}}\right)\right\} ;
\end{aligned}
$$

see the Appendix (p. 553) for the proof. We can interpret the resulting procedure as an instance of the hierarchical clustering algorithm of Ward (1963), as the parameter $\theta$ can be regarded as an analogue of the presumed number of clusters in the classical Ward procedure. For any given $\theta$, we can compute functionals of the prior information we are assigning to partitions through simulation.

Next we take $m\left(\mathbf{y}_{C_{j}}\right)$ to be (2.8). The procedure works as follows. Iteratively, we combine two clusters $C_{j_{1}}$ and $C_{j_{2}}$ if

$$
\sum_{i_{1} \in C_{j_{1}}} \sum_{i_{2} \in C_{j_{2}}} d_{i_{1}, i_{2}} \leq \min _{j_{2}, j_{1} \in\{1, \ldots, n(\mathbf{p})\}, j_{1} \neq j_{2}}\left\{\sum_{i_{1} \in C_{j_{1}}} \sum_{i_{2} \in C_{j_{2}}} d_{i_{1}, i_{2}}\right\} .
$$

For the regression case, we take $m\left(\mathbf{y}_{C_{j}}\right)$ to be (2.9), and the procedure then works as follows:

$$
\begin{aligned}
& \left(\hat{\boldsymbol{\beta}}_{C_{j_{1}}}-\hat{\boldsymbol{\beta}}_{C_{j_{2}}}\right)^{\prime} \mathbf{W}_{C_{j_{1}}, C_{j_{2}}}\left(\hat{\boldsymbol{\beta}}_{C_{j_{1}}}-\hat{\boldsymbol{\beta}}_{C_{j_{2}}}\right) \\
& \quad \leq \min _{j_{2}, j_{1} \in\{1, \ldots, n(\mathbf{p})\}, j_{1} \neq j_{2}}\left\{\left(\hat{\boldsymbol{\beta}}_{C_{j_{1}}}-\hat{\boldsymbol{\beta}}_{C_{j_{2}}}\right)^{\prime} \mathbf{W}_{C_{j_{1}}, C_{j_{2}}}\left(\hat{\boldsymbol{\beta}}_{C_{j_{1}}}-\hat{\boldsymbol{\beta}}_{C_{j_{2}}}\right)\right\},
\end{aligned}
$$

where

$$
\mathbf{W}_{C_{j_{1}}, C_{j_{2}}}=\left[\left(\mathbf{X}_{C_{j_{1}}}^{\prime} \mathbf{X}_{C_{j_{1}}}\right)^{-1}+\left(\mathbf{X}_{C_{j_{2}}}^{\prime} \mathbf{X}_{C_{j_{2}}}\right)^{-1}\right]^{-1}
$$

\subsection{Real ANd Simulated Datasets}

In order to make comparisons with different procedures, we employ four sets of artificial data and two sets of real data to illustrate our methodology.

\subsubsection{Simulated Mixtures}

We generate four sets of data. We first consider a four-component mixture of bivariate Normal densities,

$$
\begin{aligned}
& \sum_{i=1}^{4} w_{i} N\left(\mu_{i}, \Sigma_{i}\right) \text { with parameters } \\
& \mu_{1}=(2,2)^{\prime} \quad \mu_{2}=(2,-2)^{\prime} \quad \mu_{3}=(-2,2)^{\prime} \quad \mu_{4}=(-2,-2)^{\prime} \\
& \Sigma_{1}=\mathbf{I}_{2 \times 2} \quad \Sigma_{2}=\mathbf{I}_{2 \times 2} \quad \Sigma_{3}=\mathbf{I}_{2 \times 2} \quad \Sigma_{4}=\mathbf{I}_{2 \times 2},
\end{aligned}
$$


where $\mathbf{I}_{2 \times 2}$ represent the identity matrix of order 2 . We generate an artificial dataset with the following settings,

$\begin{array}{ccccc} & w_{1} & w_{2} & w_{3} & w_{4} \\ \text { Model 1 } & 1 & 0 & 0 & 0 \\ \text { Model 2 } & 1 / 2 & 1 / 2 & 0 & 0 \\ \text { Model 3 } & 1 / 3 & 1 / 3 & 1 / 3 & 0 \\ \text { Model 4 } & 1 / 4 & 1 / 4 & 1 / 4 & 1 / 4\end{array}$

We take the covariate $\left[\mathbf{x}_{1} \ldots \mathbf{x}_{S}\right]^{\prime}=\mathbf{I}_{2 \times 2}$ for $S=2$ and $K=2$. A comparison between the stochastic search procedures and hierarchical clustering procedures is performed. We take 100 sets of data from each of the models $1-4$, each with $n=100$ data points. We fit the data to the model (2.5) with the Dirichlet process prior. The parameters are assigned to be $\theta=1, a_{0}=1, b_{0}=0.01, \mathbf{m}_{0}=[0 \ldots 0]^{\prime}, \mathbf{t}_{0}=0.01 \mathbf{I}$. We employ both SS and BH procedures. For the stochastic search method, we run the Gibbs sampler for 20,000 sweeps. We record the partition of greatest posterior probability among all 20,000 sweeps. In Figure 1 we plot the $\log (\phi(\mathbf{p}))$ of the stochastic search (SS) versus that of Bayesian hierarchical clustering procedures $(\mathrm{BH})$. This seems to suggest that the "hidden" number of components has an effect on the performance of both procedures. The stochastic search (SS) attains higher posterior probability partitions than the Bayesian hierarchical clustering procedure $(\mathrm{BH})$ when the "hidden" number of components increases.

\subsubsection{Galaxy Data}

The famous Roeder (1990) galaxy data consist of measurements of velocities in $\mathrm{km} / \mathrm{sec}$ of 82 galaxies from a survey of the Corona Borealis region. The dataset is available in the article, as well as in the VR package of the statistical system R. We take the covariate $\left[\mathbf{x}_{1} \cdots \mathbf{x}_{S}\right]^{\prime}=1$ for $S=1$ and $K=1$. We fit our models to these data. We plot the $\log$ posterior probability $\log (\phi(\mathbf{p}))$ against sweep number for the first 200 sweeps of the stochastic search (SS) in Figure 2, and against the number of clusters of the Bayesian hierarchical clustering procedures $(\mathrm{BH})$ in Figure 3. Interestingly, both procedures attain the same highest-posterior-probability $\mathbf{p}$, suggesting that the optimal number of clusters is three. Moreover, stochastic search (SS) first attains the best $\mathbf{p}$ at the 181th sweep of the 20,000 sweeps used for comparison. Of course, we may want more sweeps to get better performance.

In Figure 4, in addition to the histogram we indicate the observations, and the inferred clusters, numbered in no particular order.

\subsubsection{Leukemia Data}

The leukemia data of Golub et al. (1999) consist of 7,129 gene expression levels of 72 patients with either acute myeloid leukemia (AML) or acute lymphoblastic leukemia (ALL). Golub et al. (1999) split the data into training and test sets. The training set consists of 38 patients, of which 27 have acute lymphoblastic (ALL) and 11 have acute myeloid leukemia (AML) cases, while the test set of 34 patients has 20 with ALL and 14 with 

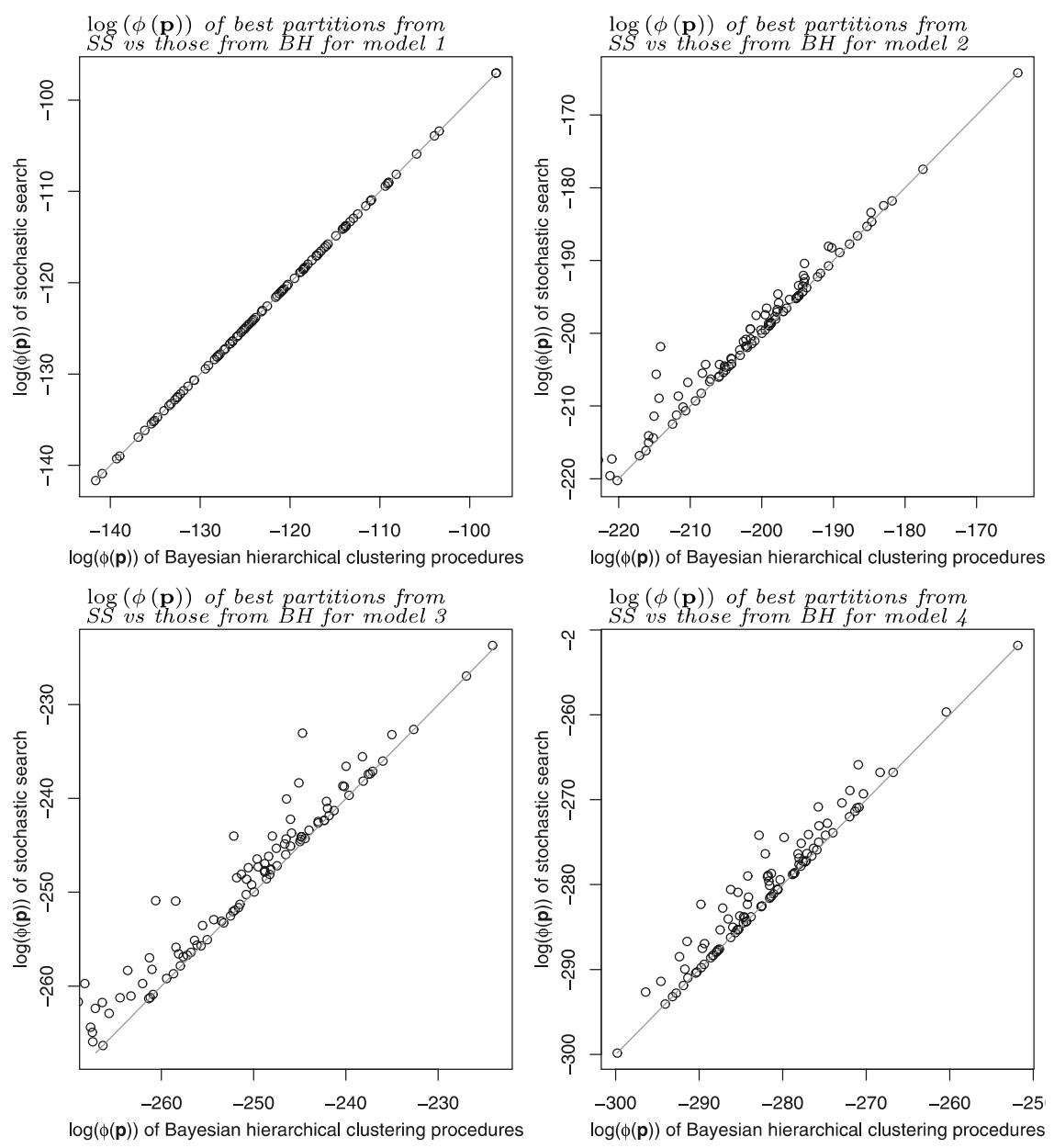

Figure 1. Comparisons between SS and BH under models 1-4 based on log posterior probability.

AML. Acute lymphoblastic leukemia actually arises from two different types of lymphocytes (T-cell and B-cell). Among all 47 ALL patients, there are 38 and 9 with Bcell and T-cell lymphocytes respectively. The dataset is available at the Web site http: $/ / w w w$-genome.wi.mit.edu/mpr/data_set_ALL_AML.html and in the golubEsets package of the Bioconductor system (Gentleman et al. 2004). Following Dudoit et al. (2002), to transform the data we employ: (a) thresholding using a floor of 100 and ceiling of 16,000 ; (b) filtering, exclusion of genes with $\max / \min \leq 5$ or $\max -\min \leq 1,600$, where max and min refer to the maximum and minimum intensities for a particular gene across the 72 mRNA samples; and (c) base 10 logarithmic transformation. There are 1,656 genes left. The analyzed dataset is a normalized version of the selected gene expressions. Each gene is normalized by subtracting its mean and dividing by its standard deviation across 


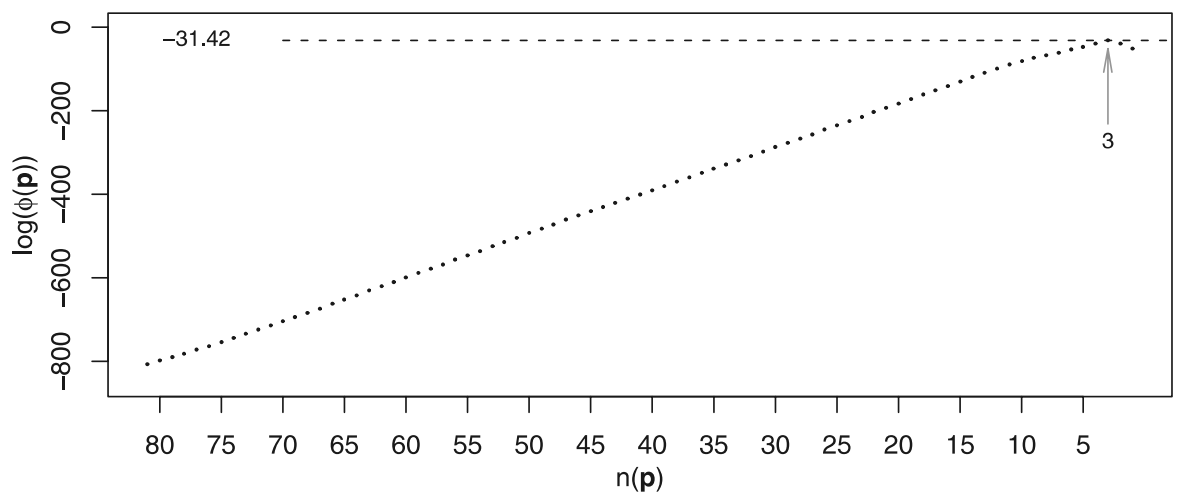

Figure 2. Log posterior partition probability versus sweep number, using SS algorithm applied to the galaxy data.

patients/samples. The design matrix of covariates $\left[\mathbf{x}_{1} \ldots \mathbf{x}_{S}\right]^{\prime}$ is taken to be

$$
\left[\mathbf{x}_{1} \ldots \mathbf{x}_{S}\right]^{\prime}=\underbrace{\left[\begin{array}{lll}
1 \ldots 1 & 1 \ldots 1 & 1 \ldots 1 \\
1 \ldots 1 & 0 \ldots 0 & 0 \ldots 0 \\
0 \ldots 0 & 1 \ldots 1 & 0 \ldots 0
\end{array}\right]^{\text {ALL (T-CELL) }}}_{\text {ALL (B-CELL) }}
$$

for $S=72$ and $K=3$.

We fit the leukemia data to the cluster models. We plot the log posterior probability $\log (\phi(\mathbf{p}))$ against the number of clusters of the Bayesian hierarchical clustering procedures (BH) in Figure 5, and against sweep number for 20,000 sweeps of the stochastic search (SS) in Figure 6. In the plots, we also indicate where the best partitions are. In this case, stochastic search (SS) performs much better than Bayesian hierarchical clustering procedures $(\mathrm{BH})$ in terms of quality, in that it discovers higher posterior probability partitions.

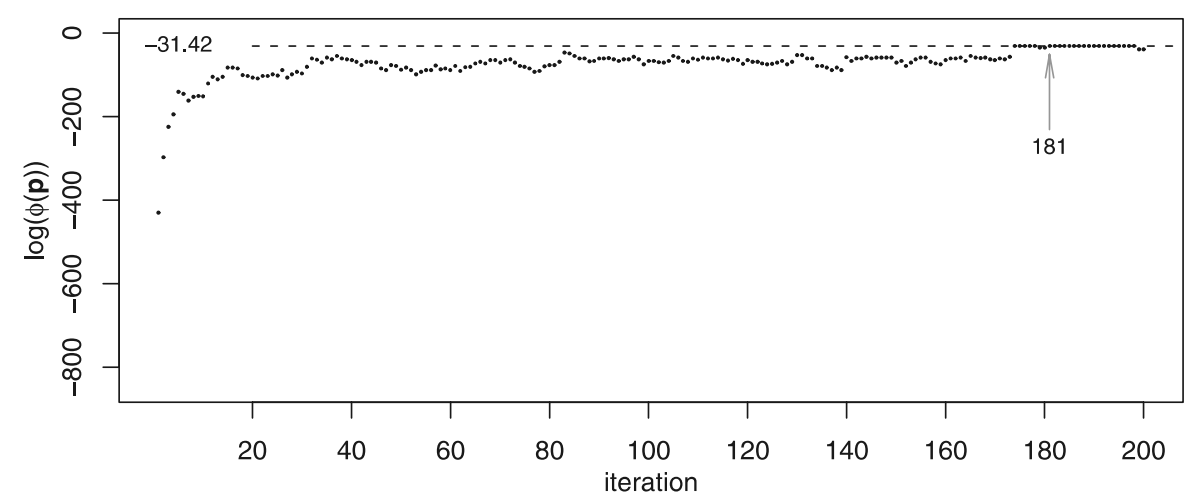

Figure 3. Log posterior partition probability versus number of clusters, using BH algorithm applied to the galaxy data. 


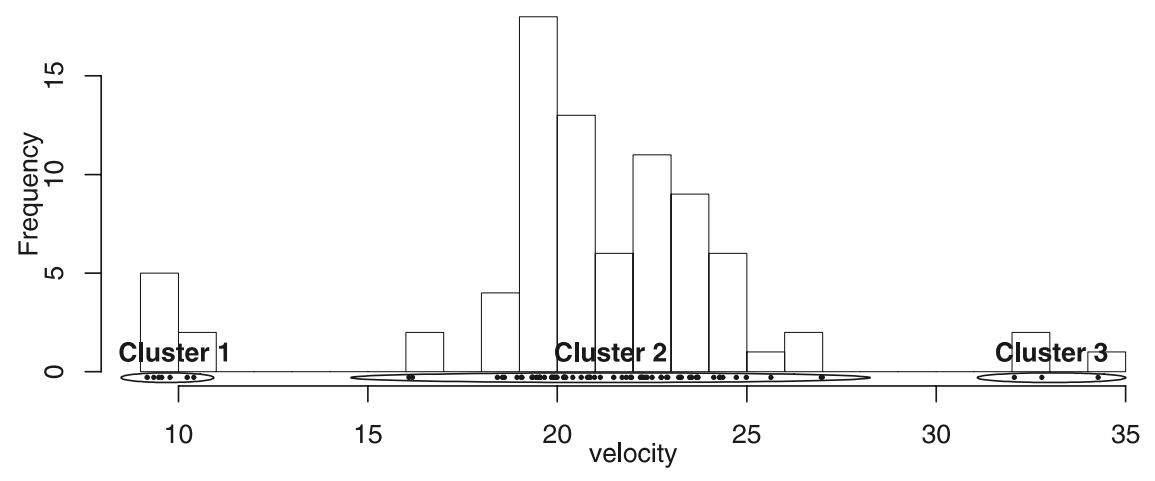

Figure 4. The best partition of the galaxy data produced by the SS and BH algorithms.

\section{OPTIMAL BAYESIAN CLUSTERING USING A PAIRWISE COINCIDENCE LOSS FUNCTION}

As previously commented, the maximum a posteriori partition has no objective status as a best estimate of the clustering of the data. Posterior modes can be increasingly unrepresentative measures of the "center" of a posterior distribution as the dimensionality of the unknown parameter increases. In normative Bayesian theory, point estimation can only be accomplished after specifying a loss function: the optimal estimate is that parameter value minimizing the posterior expected loss. That is, if the focus is on the partition $\mathbf{p}$, we have to define a loss function $L(\mathbf{p}, \widehat{\mathbf{p}})$, the cost incurred in declaring that the true partition is $\widehat{\mathbf{p}}$ when it is really $\mathbf{p}$, and choose as our optimal partition that $\widehat{\mathbf{p}}$ which minimizes $E(L(\mathbf{p}, \widehat{\mathbf{p}}) \mid \mathbf{y})$. Of course, the partition which maximizes the posterior probability is the optimal Bayesian estimator under zero-one loss; however, we regard this as very weak justification for using it as we do not find this loss function intuitively appealing.

The requirements of exchangeability of subset labels and items imposes strong con-

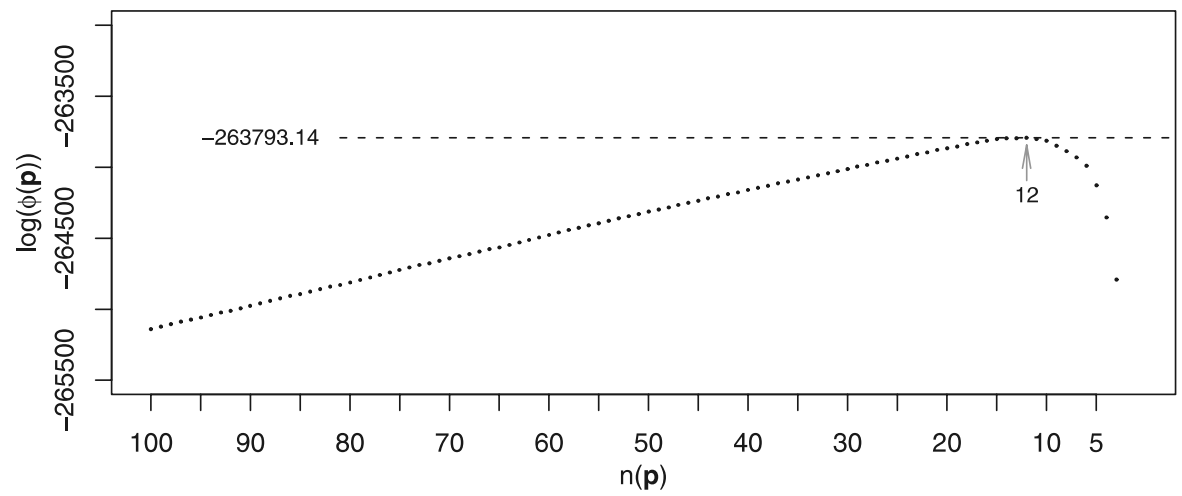

Figure 5. Log posterior partition probability versus number of clusters, using BH algorithm applied to the leukemia data. 


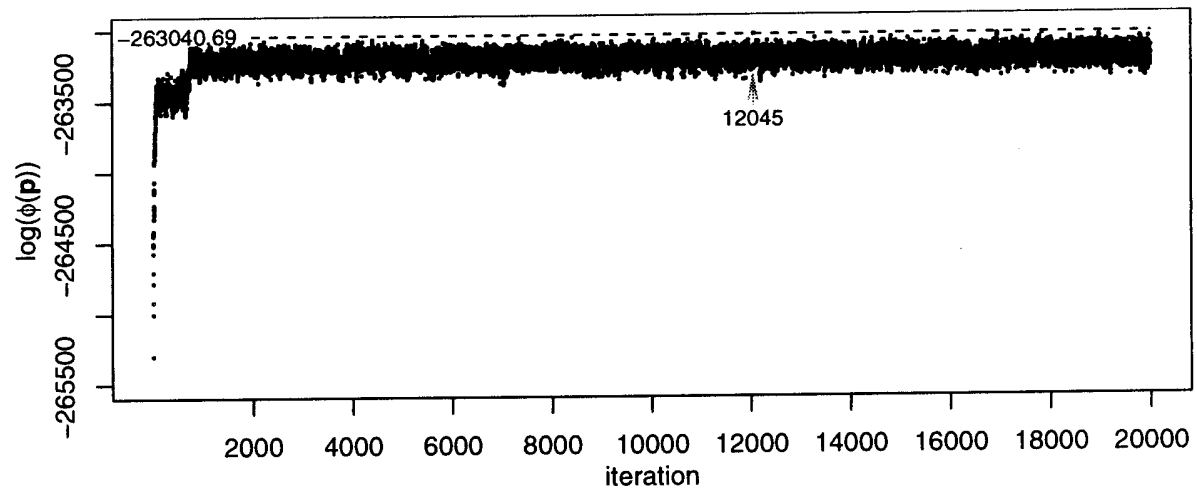

Figure 6. Log posterior partition probability versus sweep number, using SS algorithm applied to the leukemia data.

straints on the form of $L(\cdot, \cdot)$, and the interests of tractability are even more demanding. We choose to use one of the loss functions discussed by Binder $(1978,1981)$, which considers pairs of items and incurs a penalty for each pair that are clustered together when they should not be, and vice versa. That is, if $c_{i}$ denotes the allocation variable: $c_{i}=k$ if and only if $i \in C_{k}$, we define

$$
L(\mathbf{p}, \widehat{\mathbf{p}})=\sum_{(i, j) \in \mathcal{M}}\left(a \mathbb{I}\left[c_{i}=c_{j}, \widehat{c}_{i} \neq \widehat{c}_{j}\right]+b \mathbb{I}\left[c_{i} \neq c_{j}, \widehat{c}_{i}=\widehat{c}_{j}\right]\right) .
$$

Here $\mathcal{M}=\{(i, j): i<j ; i, j \in\{1, \ldots, n\}\}$, and $a$ and $b$ are given nonnegative constants, the unit costs for each kind of pairwise misclassification.

The posterior expected loss is evidently

$$
E(L(\mathbf{p}, \widehat{\mathbf{p}}) \mid \mathbf{y})=\sum_{(i, j) \in \mathcal{M}}\left(a \mathbb{I}\left[\widehat{c}_{i} \neq \widehat{c}_{j}\right] P\left\{c_{i}=c_{j} \mid \mathbf{y}\right\}+b \mathbb{I}\left[\widehat{c}_{i}=\widehat{c}_{j}\right] P\left\{c_{i} \neq c_{j} \mid \mathbf{y}\right\}\right) .
$$

Let us abbreviate the posterior coincidence probabilities $P\left\{c_{i}=c_{j} \mid \mathbf{y}\right\}=\rho_{i j}$, then we can write

$$
E(L(\mathbf{p}, \widehat{\mathbf{p}}) \mid \mathbf{y})=a \sum_{(i, j) \in \mathcal{M}} \rho_{i j}-(a+b) \sum_{(i, j) \in \mathcal{M}} \mathbb{I}\left[\widehat{c}_{i}=\widehat{c}_{j}\right]\left(\rho_{i j}-K\right),
$$

where $K=b /(a+b) \in[0,1]$. Minimizing the posterior expected loss is thus equivalent to maximizing

$$
\ell(\widehat{\mathbf{p}}, K)=\sum_{(i, j) \in \mathcal{M}} \mathbb{I}\left[\widehat{c}_{i}=\widehat{c}_{j}\right]\left(\rho_{i j}-K\right)
$$

over choice of $\widehat{\mathbf{p}}$.

Curves of the objective function $\ell(\widehat{\mathbf{p}}, K)$ regarded as a function of $K$ are linear and have nonpositive integer slopes and nonnegative intercepts, for each $\widehat{\mathbf{p}}$. These functions of $K$ characterize the quality of each possible $\widehat{\mathbf{p}}$, and the whole ensemble of such functions determines in particular for which $K$, if any, each partition is optimal, as well as defining the optimal $\widehat{\mathbf{p}}$ for each $K$. Our approach, therefore, is to consider all values of $K$ simultaneously. 


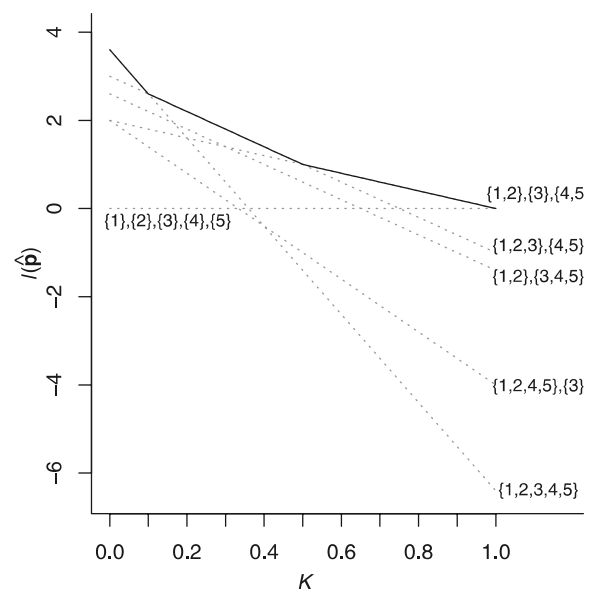

Figure 7. The objective function $\ell(\hat{\mathbf{p}}, K)$ versus $K$ for various partitions, for the illustrative example.

Example. Suppose there are five items and that the partitions of these have probabilities

$$
\begin{array}{ll}
\mathbf{p}_{1}=\{\{1,2,3\},\{4,5\}\} & \mathbb{P}\left\{\mathbf{p}=\mathbf{p}_{1}\right\}=0.5 \\
\mathbf{p}_{2}=\{\{1,2\},\{3\},\{4,5\}\} & \mathbb{P}\left\{\mathbf{p}=\mathbf{p}_{2}\right\}=0.2 \\
\mathbf{p}_{3}=\{\{1,2\},\{3,4,5\}\} & \mathbb{P}\left\{\mathbf{p}=\mathbf{p}_{3}\right\}=0.3 .
\end{array}
$$

The $\rho$ matrix can be calculated easily based on those probabilities.

$\begin{array}{cccccc} & 1 & 2 & 3 & 4 & 5 \\ 1 & - & 1 & 0.5 & 0 & 0 \\ 2 & - & - & 0.5 & 0 & 0 \\ 3 & - & - & - & 0.3 & 0.3 \\ 4 & - & - & - & - & 1 \\ 5 & - & - & - & - & -\end{array}$

Figure 7 shows the objective function $\ell(\widehat{\mathbf{p}}, K)$ and related partitions. The dotted lines represent the performance of various partitions. The solid curves represent the optimal performance for each value of $K$. It is easy to observe that the solid curve is formed from three straight line segments. Each of these segments corresponds to a partition that is optimal for certain $K$; note that one of these three has zero posterior probability, while one of those with positive posterior probability is never optimal.

\subsection{A binary Integer Programming Formulation}

We will represent the maximization of (4.1) as a binary integer programming problem. Let us replace the indicator function $\mathbb{I}\left[\hat{c}_{i}=\hat{c}_{j}\right]$ by a binary $(0 / 1)$ variable $X_{i j}$; the objective function is a linear combination of the $X_{i j}$ with weights $\left(\rho_{i j}-K\right)$. Since $\hat{c}$ represents a proper partition of the items, the $X_{i j}$ are subject to constraints. It is easy to see that what we require is that for all triples $(i, j, k)$, if $X_{i j}=1$, then $X_{i k}=X_{j k}$. These boolean constraints can be represented as algebraic inequalities, and we find that maximization of 


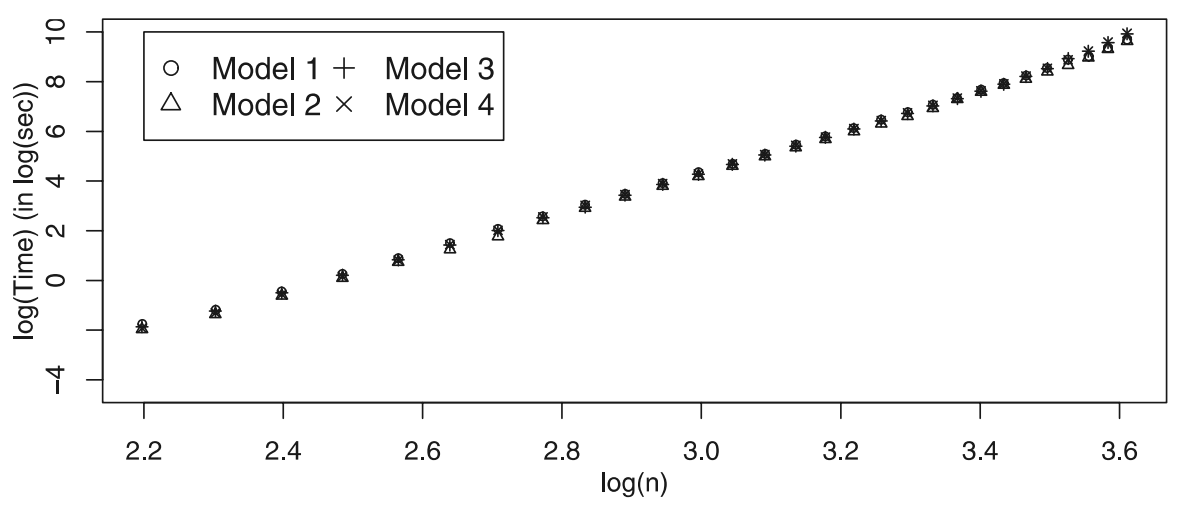

Figure 8. Running time versus problem size for simulated mixture models $1-4$, using linear programming.

(4.1) is equivalent to solving the optimization problem:

$$
\begin{aligned}
\max _{\left\{X_{i j}: i, j \in \mathcal{M}\right\}} & \sum_{i, j \in \mathcal{M}} X_{i j}\left(\rho_{i j}-K\right) \\
\text { s.t. } & X_{i j}+X_{i k}-X_{j k} \leq 1 \text { for }\{i \neq j \neq k \neq i: i, j, k \in\{1, \ldots, n\}\} \\
& X_{i j} \in\{0,1\} \quad \text { for } i, j \in \mathcal{M} .
\end{aligned}
$$

We conduct a modest experiment to study the feasibility of solving this programming problem, although Bansal et al. (2004) already showed that this is an NP hard problem. We use datasets of $n$ points from each of models $1-4$, for various values of $n$, each replicated 10 times. The regression marginal (2.5) is fitted to each dataset, using a Dirichlet process prior with settings $\theta=1.0, a_{0}=1.0, b_{0}=0.01, m_{0}=\mathbf{0}, t_{0}=0.01 \mathbf{I}$. The matrix $\left[\mathbf{x}_{1} \ldots \mathbf{x}_{S}\right]^{\prime}$ is chosen to be an identity matrix $\mathbf{I}_{S \times S}$. The MCMC sampler discussed in Section 3.1 was used to generate 10,000 sweeps following 10,000 burn-in for each data sequence. We then estimated $\rho_{i j}$ for each data sequence using the last 10,000 sweeps. We use the lpSolve package (Berkelaar et al. 2006) of the statistical system $R$ to solve the programming problem (4.2) for each $K \in\{0.1,0.2,0.3,0.4,0.5,0.6,0.7,0.8,0.9\}$. The code in this package solves general mixed linear-integer programmes. In all of the computations using lpSolve reported in this article, exactly the same solutions are obtained whether or not the solution is constrained to be integer; however, it is possible to construct sets of $\rho_{i j}$ for which this is not true. The times spent on solving the problem are recorded in seconds. In Figure 8 we plot $\log$ (Time) against $\log (n)$ for each dataset and least square regression parameters are estimated. In our very rough prediction, we may spend 225.57 billion years to solve a programming program with $n=1,000$. This demonstrates the need for a faster algorithm to perform the optimization, or at least to obtain an approximation to the optimum.

\subsection{A Novel Algorithm}

When $K=1$ or $K=0$, the optimal partitions are trivially obtained-they are those corresponding to $X_{i j}=0$ and $X_{i j}=1$ for all $i, j$ in the set $\mathcal{M}$, respectively. For other $K$, as 
we have seen, the solution is not obvious and the problem NP hard. We propose an iterative procedure that gives a locally optimal solution for the problem. The main idea is to solve the problem partially in each iteration; see the following algorithm 1 . We subsequently introduce a procedure that gives locally optimal solutions simultaneously for all $K \in[0,1]$; see Algorithm 2.

Algorithm 1. Define $\mathcal{M}_{i}=\{(1, i), \ldots,(i-1, i),(i, i+1), \ldots,(i, n)\}$. Given a value $K$ and a partition $\mathbf{p}$,

Step 1. For $i=1, \ldots, n$, given $\left\{X_{j k}: j, k \in \mathcal{M} \backslash \mathcal{M}_{i}\right\}$ are fixed, solve a binary integer programming problem

$$
\begin{aligned}
\max _{\left\{X_{j k}: j, k \in \mathcal{M}_{i}\right\}} & \sum_{j, k \in \mathcal{M}_{i}} X_{j k}\left(\rho_{j k}-K\right) \\
\text { s.t. } & X_{i j}+X_{i k}-X_{j k} \leq 1 \text { for }\{i \neq j \neq k \neq i: i, j, k \in\{1, \ldots, n\}\} \\
& X_{i j} \in\{0,1\} \text { for } i, j \in \mathcal{M}
\end{aligned}
$$

Step 2. Go to Step 1 if the value of the objective function evaluated at the new partition exceeds that on the previous iteration.

Algorithm 1 expresses the idea that we can seek to increase the objective function by cycling over items $i$, taking $i$ out of the current partition and reallocating it in the optimal way: either by assigning it to an existing cluster or by creating a new one.

As we vary $K$ and thus the relative costs of the two kinds of pairwise error in clustering, the optimal partition varies. If $K$ is not predetermined, we may repeat Algorithm 1 for several choices of $K$, but there are advantages of convenience and efficiency in considering all $K \in[0,1]$ simultaneously. Empirically, we find this also helps to avoid converging to local optima.

As we see from (4.2), each partition $\mathbf{p}$ is characterized by a nonincreasing linear function of $K$, with intercept and slope depending on $\mathbf{p}$. For any set of partitions $\mathcal{P}$, we can define

$$
\ell(\mathcal{P}, K)=\max _{\mathbf{p} \in \mathcal{P}} \ell(\mathbf{p}, K)
$$

and by basic properties of convexity, this is a nonincreasing convex polygonal (piecewise linear) function of $K$ for each $\mathcal{P}$, and is nondecreasing (with respect to set inclusion) in $\mathcal{P}$ for each $K$. Further, $\ell(\mathcal{P}, K)$ can be parameterized by a set of increasing values $0=K_{0}<$ $K_{1}<\cdots<K_{r}=1$ and partitions $\mathbf{p}_{1}, \mathbf{p}_{2}, \ldots, \mathbf{p}_{r} \in \mathcal{P}$, with

$$
\ell(\mathcal{P}, K)=\ell\left(\mathbf{p}_{s}, K\right) \quad \text { for all } \quad K \in\left[K_{s-1}, K_{s}\right]
$$

for $s=1,2, \ldots, r$. Note that $\mathbf{p}_{s}$ is optimal among $\mathbf{p} \in \mathcal{P}\left(\ell\left(\mathbf{p}_{s}, K\right) \geq \ell(\mathbf{p}, K)\right)$ for all $K \in\left[K_{s-1}, K_{s}\right]$.

Our true objective is, for every $K$, to maximize $\ell(\mathbf{p}, K)$ over the set $\widetilde{\mathcal{P}}$ of all partitions; we could then use (4.3) with $\mathcal{P}=\widetilde{\mathcal{P}}$ to read off the optimal partition (which is evidently constant on intervals of $K$ ) for each $K$. For any subset $\mathcal{P} \subseteq \widetilde{\mathcal{P}}, \ell(\mathcal{P}, K)$ is a lower bound 


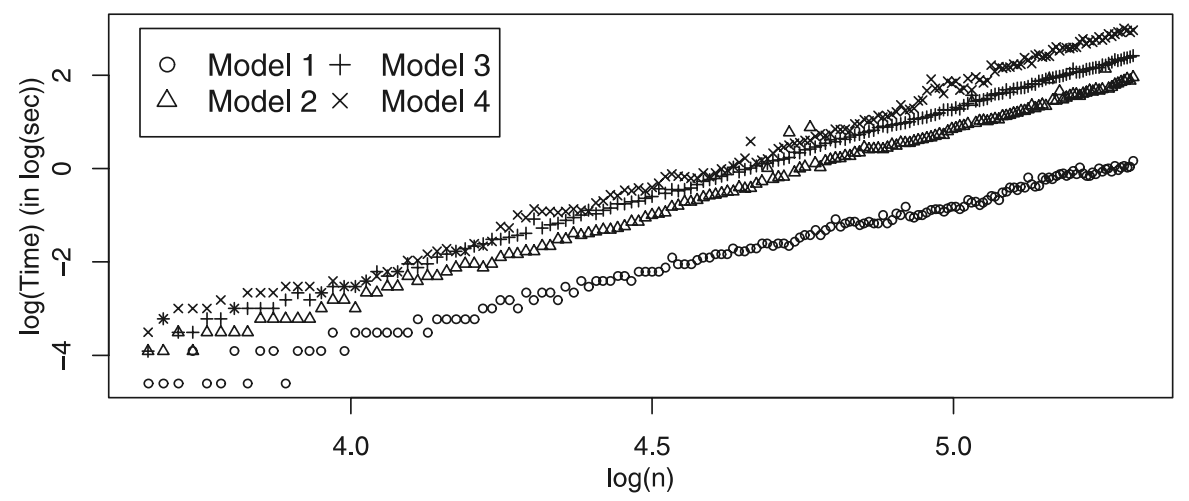

Figure 9. Running time versus problem size for simulated mixture models 1-4, using Algorithm 2.

for $\ell(\widetilde{\mathcal{P}}, K)$. Algorithm 2 is a heuristic for iteratively increasing $\mathcal{P}$ and hence the lower bound $\ell(\mathcal{P}, K)$ to obtain successive approximations to the optimum.

Algorithm 2. Initiate the algorithm by setting $\mathcal{P}=\left\{\mathbf{p}_{a}, \mathbf{p}_{s}\right\}$, where $\mathbf{p}_{a}$ is the partition that has all items in one cluster and $\mathbf{p}_{s}$ is the partition consisting of all singleton clusters.

Step 1. Take a pair of adjacent partitions, say $\mathbf{p}_{s}$ and $\mathbf{p}_{s+1}$, from the set representing $\ell(\mathcal{P}, K)$ defined in (4.3).

Step 2. Run Algorithm 1 twice using the value $K_{s}$, starting from partitions $\mathbf{p}_{s}$ and $\mathbf{p}_{s+1}$. The resulting partitions are $\mathbf{p}_{s}^{*}$ and $\mathbf{p}_{s+1}^{*}$.

Step 3. Insert $\mathbf{p}_{s}^{*}$ and $\mathbf{p}_{s+1}^{*}$ into the set $\mathcal{P}$.

Step 4. Recompute the representation (4.3) for the modified set of partitions $\mathcal{P}$.

Step 5. End if all pairs $\left(\mathbf{p}_{s}, \mathbf{p}_{s+1}\right)$ have been visited and the representation of the set $\mathcal{P}$ is unchanged; go to step 1 otherwise.

We perform an experiment based on the same settings as in the previous section, using Algorithm 2 on the interval [0, 0.99]. (We find that the optimal partition changes rapidly for $K$ near 1, so that Algorithm 2 is computationally expensive applied to the full interval.) In Figure 9 we plot the $\log$ (Time) against $\log (n)$ for each dataset, and estimate the least squares regression. Interestingly, the run time of the algorithm is an increasing function of the number of hidden components. We predict a run time of 3.51 hours to solve the optimisation for $n=1,000$ items under model 4 .

As pointed out by a referee, we may also attempt to find the optimal partition using stochastic search: that is, to deliver as an estimate the partition with smallest expected loss encountered during the Monte Carlo run. This is somewhat similar to the approach of Dahl (2006), in a different model, although he did not use an explicit decision theoretic formulation. We have not explored this, but note that this method always yields one of the partitions actually visited during the run, whereas our approach considers a much larger set of possible partitions. 


\subsection{NUMERICAL EXPERIMENTS}

\subsubsection{Assessment of Heuristic Approximation}

Our assessment of the performance of the algorithm uses models 1-4, but with small datasets, $n$ varying from 3 to 15 . Ten thousand replications are made of each case. We fit the regression marginal (2.5) to each dataset, with the Dirichlet process prior. The matrix $\left[\mathbf{x}_{1} \ldots \mathbf{x}_{S}\right]^{\prime}$ is chosen to be the identity matrix $\mathbf{I}_{S \times S}$. The sampling uses the Gibbs sampler with parameters $\theta=1.0, a_{0}=1.0, b_{0}=0.01, m_{0}=\mathbf{0}, t_{0}=0.01 \mathbf{I}$ and generates 10,000 partitions following 10,000 burn-in for each dataset. We estimate $\rho_{i j}$ for each dataset based on the last 10,000 sweeps. We use our Algorithm 2 to generate best partitions for $K \in$ $[0,0.99]$, and compare these to the true optima, calculated using 1 pSolve as in Section 4.1. Tables 1-4 show the proportions of runs in which the algorithm finds the true optimum for different $K$ and $n$, while Tables 5-8 show the mean of the ratios of the optimized $\ell(\mathbf{p}, K)$ for different $K$ and $n$.

\subsubsection{Optimal versus Maximum Probability Partitions}

To get some appreciation of the difference between (approximate) MAP partitions and (approximate) optimal partitions according to our loss function, we make some comparisons of point estimates based on three different approaches, stochastic search (SS), Bayesian hierarchical clustering procedure (BH), and the loss function (Algorithm 2), using simulated data from models 1-4. Similarly to the simulation study in Section 3.3.1, we take 100 sets of data from each of the models $1-4$, each with $n=100$ data points. We fit the model (2.5) with the Dirichlet process prior. The parameters are assigned to be $\theta=1, a_{0}=1, b_{0}=0.01, \mathbf{m}_{0}=[0 \ldots 0]^{\prime}, \mathbf{t}_{0}=0.01 \mathbf{I}$.

For the stochastic search method, we run the Gibbs sampler for 20,000 sweeps. We record the partition of greatest posterior probability among all 20,000 sweeps. We then use Algorithm 2 based on the last 10,000 MCMC samples, following 10,000 burn-in to produce the $\rho$ matrix. We run Algorithm 2 and the $\ell$ functions (defined by Equation (4.1)) to get the point estimates for $K \in\{0.1,0.2, \ldots, 0.9\}$. Figure 10 (p. 550) compares log posterior probabilities $\log (\phi(\mathbf{p}))$ for the partitions produced by the three procedures, stochastic search (SS), Bayesian hierarchical clustering procedure (BH), and the loss function method (Algorithm 2). As expected, the results show that the approximate MAP methods tend to yield higher probability partitions, with SS outperforming BH on average. However, to an extent increasing with the number of components in the simulation of the data, the loss function approach nevertheless produces higher probability partitions for a substantial proportion of the datasets.

On the other hand, Figure 11 (p. 551) compares the quantity $\ell(\mathbf{p}, K)$ for the partitions produced by the three procedures. The results show that the loss function method (Algorithm 2) always yields partitions with lower posterior expected loss than the approximate MAP methods, particularly for larger values of $K$. Among the methods inferior by this criterion, $\mathrm{SS}$ is again slightly superior to $\mathrm{BH}$, although for smaller values of $K$, all three methods perform very similarly. 
Table 1. Proportion of 10,000 replicates from model 1 for which Algorithm 2 obtains the true optimum, for a range of $K$ and $n$.

\begin{tabular}{cccccccccc}
\hline \hline & $K=0.1$ & $K=0.2$ & $K=0.3$ & $K=0.4$ & $K=0.5$ & $K=0.6$ & $K=0.7$ & $K=0.8$ & $K=0.9$ \\
\hline$n=3$ & 1 & 1 & 1 & 1 & 1 & 1 & 1 & 1 & 1 \\
$n=4$ & 1 & 1 & 1 & 1 & 1 & 1 & 1 & 1 & 1 \\
$n=5$ & 1 & 1 & 1 & 1 & 1 & 1 & 1 & 1 & 0.9999 \\
$n=6$ & 0.9990 & 0.9993 & 0.9994 & 0.9998 & 0.9996 & 0.9994 & 0.9996 & 0.9998 & 0.9998 \\
$n=7$ & 0.9985 & 0.9983 & 0.9984 & 0.9985 & 0.9984 & 0.9986 & 0.9993 & 0.9993 & 0.9995 \\
$n=8$ & 0.9971 & 0.9974 & 0.9982 & 0.9986 & 0.9982 & 0.9980 & 0.9982 & 0.9991 & 0.9991 \\
$n=9$ & 0.9966 & 0.9978 & 0.9978 & 0.9966 & 0.9977 & 0.9979 & 0.9981 & 0.9990 & 0.9988 \\
$n=10$ & 0.9986 & 0.9980 & 0.9983 & 0.9987 & 0.9983 & 0.9984 & 0.9985 & 0.9991 & 0.9984 \\
$n=11$ & 0.9983 & 0.9986 & 0.9987 & 0.9985 & 0.9987 & 0.9982 & 0.9979 & 0.9982 & 0.9982 \\
$n=12$ & 0.9987 & 0.9981 & 0.9983 & 0.9978 & 0.9987 & 0.9986 & 0.9993 & 0.9993 & 0.9984 \\
$n=13$ & 0.9989 & 0.9987 & 0.9987 & 0.9986 & 0.9990 & 0.9987 & 0.9986 & 0.9991 & 0.9982 \\
$n=14$ & 0.9985 & 0.9993 & 0.9996 & 0.9990 & 0.9986 & 0.9989 & 0.9991 & 0.9985 & 0.9987 \\
$n=15$ & 0.9992 & 0.9997 & 0.9997 & 0.9992 & 0.9994 & 0.9993 & 0.9997 & 0.9987 & 0.9984 \\
\hline
\end{tabular}

Table 2. Proportion of 10,000 replicates from model 2 for which Algorithm 2 obtains the true optimum, for a range of $K$ and $n$.

\begin{tabular}{cccccccccc}
\hline \hline & $K=0.1$ & $K=0.2$ & $K=0.3$ & $K=0.4$ & $K=0.5$ & $K=0.6$ & $K=0.7$ & $K=0.8$ & $K=0.9$ \\
\hline$n=3$ & 1 & 1 & 1 & 1 & 1 & 1 & 1 & 1 & 1 \\
$n=4$ & 1 & 1 & 1 & 1 & 1 & 1 & 1 & 1 & 1 \\
$n=5$ & 1 & 1 & 1 & 1 & 1 & 1 & 1 & 1 & 1 \\
$n=6$ & 0.9978 & 0.9996 & 0.9999 & 0.9998 & 0.9998 & 0.9998 & 0.9996 & 0.9997 & 0.9995 \\
$n=7$ & 0.9871 & 0.9952 & 0.9983 & 0.9995 & 0.9998 & 0.9996 & 0.9997 & 0.9995 & 0.9993 \\
$n=8$ & 0.9748 & 0.9875 & 0.9945 & 0.9972 & 0.9989 & 0.9995 & 0.9995 & 0.9993 & 0.9988 \\
$n=9$ & 0.9648 & 0.9775 & 0.9904 & 0.9945 & 0.9965 & 0.9979 & 0.9988 & 0.9989 & 0.9980 \\
$n=10$ & 0.9564 & 0.9748 & 0.9875 & 0.9927 & 0.9969 & 0.9976 & 0.9983 & 0.9992 & 0.9987 \\
$n=11$ & 0.9529 & 0.9696 & 0.9852 & 0.9918 & 0.9955 & 0.9971 & 0.9967 & 0.9975 & 0.9986 \\
$n=12$ & 0.9493 & 0.9673 & 0.9829 & 0.9914 & 0.9943 & 0.9955 & 0.9970 & 0.9971 & 0.9983 \\
$n=13$ & 0.9478 & 0.9688 & 0.9838 & 0.9899 & 0.9922 & 0.9943 & 0.9950 & 0.9961 & 0.9973 \\
$n=14$ & 0.9517 & 0.9702 & 0.9825 & 0.9902 & 0.9926 & 0.9932 & 0.9951 & 0.9965 & 0.9962 \\
$n=15$ & 0.9475 & 0.9689 & 0.9836 & 0.9877 & 0.9928 & 0.9937 & 0.9959 & 0.9955 & 0.9968 \\
\hline
\end{tabular}


Table 3. Proportion of 10,000 replicates from model 3 for which Algorithm 2 obtains the true optimum, for a range of $K$ and $n$.

\begin{tabular}{cccccccccc}
\hline \hline & $K=0.1$ & $K=0.2$ & $K=0.3$ & $K=0.4$ & $K=0.5$ & $K=0.6$ & $K=0.7$ & $K=0.8$ & $K=0.9$ \\
\hline$n=3$ & 1 & 1 & 1 & 1 & 1 & 1 & 1 & 1 & 1 \\
$n=4$ & 1 & 1 & 1 & 1 & 1 & 1 & 1 & 1 & 1 \\
$n=5$ & 1 & 1 & 1 & 1 & 1 & 1 & 1 & 1 & 1 \\
$n=6$ & 0.9970 & 0.9993 & 1 & 0.9999 & 0.9999 & 0.9999 & 0.9999 & 0.9997 & 0.9996 \\
$n=7$ & 0.9891 & 0.9961 & 0.9991 & 0.9999 & 0.9998 & 0.9998 & 0.9997 & 0.9996 & 0.9992 \\
$n=8$ & 0.9752 & 0.9904 & 0.9958 & 0.9978 & 0.9992 & 0.9995 & 0.9992 & 0.9987 & 0.9984 \\
$n=9$ & 0.9616 & 0.9817 & 0.9883 & 0.9961 & 0.9982 & 0.9987 & 0.9993 & 0.9989 & 0.9982 \\
$n=10$ & 0.9463 & 0.9690 & 0.9828 & 0.9884 & 0.9956 & 0.9975 & 0.9980 & 0.9989 & 0.9987 \\
$n=11$ & 0.9377 & 0.9620 & 0.9776 & 0.9862 & 0.9931 & 0.9962 & 0.9979 & 0.9986 & 0.9983 \\
$n=12$ & 0.9240 & 0.9582 & 0.9745 & 0.9854 & 0.9907 & 0.9943 & 0.9970 & 0.9986 & 0.9983 \\
$n=13$ & 0.9168 & 0.9537 & 0.9741 & 0.9844 & 0.9885 & 0.9919 & 0.9953 & 0.9974 & 0.9974 \\
$n=14$ & 0.9164 & 0.9522 & 0.9719 & 0.9828 & 0.9881 & 0.9897 & 0.9926 & 0.9961 & 0.9968 \\
$n=15$ & 0.9257 & 0.9517 & 0.9723 & 0.9806 & 0.9867 & 0.9897 & 0.9929 & 0.9945 & 0.9962 \\
\hline
\end{tabular}

Table 4. Proportion of 10,000 replicates from model 4 for which Algorithm 2 obtains the true optimum, for a range of $K$ and $n$.

\begin{tabular}{cccccccccc}
\hline \hline & $K=0.1$ & $K=0.2$ & $K=0.3$ & $K=0.4$ & $K=0.5$ & $K=0.6$ & $K=0.7$ & $K=0.8$ & $K=0.9$ \\
\hline$n=3$ & 1 & 1 & 1 & 1 & 1 & 1 & 1 & 1 & 1 \\
$n=4$ & 1 & 1 & 1 & 1 & 1 & 1 & 1 & 1 & 1 \\
$n=5$ & 1 & 1 & 1 & 1 & 1 & 1 & 1 & 1 & 1 \\
$n=6$ & 0.9985 & 0.9999 & 0.9998 & 0.9999 & 0.9999 & 0.9999 & 0.9999 & 0.9999 & 0.9999 \\
$n=7$ & 0.9925 & 0.9981 & 0.9991 & 0.9996 & 0.9997 & 0.9995 & 0.9994 & 0.9992 & 0.9987 \\
$n=8$ & 0.9773 & 0.9917 & 0.9959 & 0.9983 & 0.9991 & 0.9991 & 0.9987 & 0.9977 & 0.9968 \\
$n=9$ & 0.9626 & 0.9807 & 0.9894 & 0.9942 & 0.9962 & 0.9981 & 0.9984 & 0.9969 & 0.9955 \\
$n=10$ & 0.9581 & 0.9759 & 0.9831 & 0.9893 & 0.9938 & 0.9968 & 0.9978 & 0.9975 & 0.9957 \\
$n=11$ & 0.9519 & 0.9698 & 0.9790 & 0.9869 & 0.9905 & 0.9948 & 0.9958 & 0.9984 & 0.9973 \\
$n=12$ & 0.9522 & 0.9658 & 0.9755 & 0.9828 & 0.9909 & 0.9934 & 0.9954 & 0.9971 & 0.9961 \\
$n=13$ & 0.9527 & 0.9677 & 0.9780 & 0.9821 & 0.9870 & 0.9917 & 0.9939 & 0.9967 & 0.9961 \\
$n=14$ & 0.9539 & 0.9676 & 0.9804 & 0.9825 & 0.9860 & 0.9906 & 0.9921 & 0.9958 & 0.9969 \\
$n=15$ & 0.9584 & 0.9709 & 0.9782 & 0.9847 & 0.9865 & 0.9888 & 0.9945 & 0.9948 & 0.9953 \\
\hline
\end{tabular}


Table 5. Mean of the ratio of expected posterior loss calculated by Algorithm 2 to the true minimized expectation, over 10,000 replicates from model 1, for various $K$ and $n$.

\begin{tabular}{llllllllll}
\hline \hline & $K=0.1$ & $K=0.2$ & $K=0.3$ & $K=0.4$ & $K=0.5$ & $K=0.6$ & $K=0.7$ & $K=0.8$ & $K=0.9$ \\
\hline$n=3$ & 1.00000 & 1.00000 & 1.00000 & 1.00000 & 1.00000 & 1.00000 & 1.00000 & 1.00000 & 1.00000 \\
$n=4$ & 1.00000 & 1.00000 & 1.00000 & 1.00000 & 1.00000 & 1.00000 & 1.00000 & 1.00000 & 1.00000 \\
$n=5$ & 1.00000 & 1.00000 & 1.00000 & 1.00000 & 1.00000 & 1.00000 & 1.00000 & 1.00000 & 1.00000 \\
$n=6$ & 1.00032 & 1.00019 & 1.00010 & 1.00000 & 1.00005 & 1.00005 & 1.00002 & 1.00000 & 1.00000 \\
$n=7$ & 1.00036 & 1.00032 & 1.00016 & 1.00013 & 1.00008 & 1.00004 & 1.00004 & 1.00001 & 1.00003 \\
$n=8$ & 1.00058 & 1.00042 & 1.00019 & 1.00012 & 1.00011 & 1.00008 & 1.00005 & 1.00002 & 1.00001 \\
$n=9$ & 1.00084 & 1.00030 & 1.00017 & 1.00017 & 1.00014 & 1.00008 & 1.00006 & 1.00002 & 1.00002 \\
$n=10$ & 1.00057 & 1.00032 & 1.00010 & 1.00006 & 1.00007 & 1.00004 & 1.00003 & 1.00002 & 1.00001 \\
$n=11$ & 1.00041 & 1.00022 & 1.00007 & 1.00005 & 1.00005 & 1.00006 & 1.00004 & 1.00002 & 1.00002 \\
$n=12$ & 1.00043 & 1.00019 & 1.00010 & 1.00007 & 1.00005 & 1.00005 & 1.00001 & 1.00001 & 1.00001 \\
$n=13$ & 1.00015 & 1.00014 & 1.00008 & 1.00007 & 1.00004 & 1.00004 & 1.00002 & 1.00001 & 1.00002 \\
$n=14$ & 1.00022 & 1.00004 & 1.00002 & 1.00005 & 1.00004 & 1.00004 & 1.00001 & 1.00001 & 1.00001 \\
$n=15$ & 1.00010 & 1.00002 & 1.00002 & 1.00003 & 1.00001 & 1.00002 & 1.00000 & 1.00001 & 1.00001 \\
\hline
\end{tabular}

Table 6. Mean of the ratio of expected posterior loss calculated by Algorithm 2 to the true minimized expectation, over 10,000 replicates from model 2 , for various $K$ and $n$.

\begin{tabular}{llllllllll}
\hline \hline & $K=0.1$ & $K=0.2$ & $K=0.3$ & $K=0.4$ & $K=0.5$ & $K=0.6$ & $K=0.7$ & $K=0.8$ & $K=0.9$ \\
\hline$n=3$ & 1.00000 & 1.00000 & 1.00000 & 1.00000 & 1.00000 & 1.00000 & 1.00000 & 1.00000 & 1.00000 \\
$n=4$ & 1.00000 & 1.00000 & 1.00000 & 1.00000 & 1.00000 & 1.00000 & 1.00000 & 1.00000 & 1.00000 \\
$n=5$ & 1.00000 & 1.00000 & 1.00000 & 1.00000 & 1.00000 & 1.00000 & 1.00000 & 1.00000 & 1.00000 \\
$n=6$ & 1.00063 & 1.00004 & 1.00001 & 1.00000 & 1.00000 & 1.00000 & 1.00000 & 1.00000 & 1.00000 \\
$n=7$ & 1.00508 & 1.00064 & 1.00009 & 1.00003 & 1.00001 & 1.00002 & 1.00001 & 1.00004 & 1.00006 \\
$n=8$ & 1.01201 & 1.00305 & 1.00083 & 1.00023 & 1.00005 & 1.00001 & 1.00001 & 1.00002 & 1.00008 \\
$n=9$ & 1.01520 & 1.00436 & 1.00129 & 1.00052 & 1.00016 & 1.00006 & 1.00002 & 1.00005 & 1.00011 \\
$n=10$ & 1.01813 & 1.00441 & 1.00121 & 1.00036 & 1.00011 & 1.00003 & 1.00005 & 1.00003 & 1.00006 \\
$n=11$ & 1.01723 & 1.00408 & 1.00107 & 1.00037 & 1.00012 & 1.00009 & 1.00006 & 1.00005 & 1.00004 \\
$n=12$ & 1.01816 & 1.00469 & 1.00111 & 1.00038 & 1.00020 & 1.00010 & 1.00006 & 1.00005 & 1.00003 \\
$n=13$ & 1.01764 & 1.00432 & 1.00116 & 1.00047 & 1.00026 & 1.00012 & 1.00008 & 1.00005 & 1.00004 \\
$n=14$ & 1.01568 & 1.00391 & 1.00123 & 1.00048 & 1.00022 & 1.00011 & 1.00007 & 1.00004 & 1.00005 \\
$n=15$ & 1.01733 & 1.00420 & 1.00100 & 1.00044 & 1.00018 & 1.00012 & 1.00006 & 1.00004 & 1.00003 \\
\hline
\end{tabular}


Table 7. Mean of the ratio of expected posterior loss calculated by Algorithm 2 to the true minimized expectation, over 10,000 replicates from model 3, for various $K$ and $n$.

\begin{tabular}{llllllllll}
\hline \hline & $K=0.1$ & $K=0.2$ & $K=0.3$ & $K=0.4$ & $K=0.5$ & $K=0.6$ & $K=0.7$ & $K=0.8$ & $K=0.9$ \\
\hline$n=3$ & 1.00000 & 1.00000 & 1.00000 & 1.00000 & 1.00000 & 1.00000 & 1.00000 & 1.00000 & 1.00000 \\
$n=4$ & 1.00000 & 1.00000 & 1.00000 & 1.00000 & 1.00000 & 1.00000 & 1.00000 & 1.00000 & 1.00000 \\
$n=5$ & 1.00000 & 1.00000 & 1.00000 & 1.00000 & 1.00000 & 1.00000 & 1.00000 & 1.00000 & 1.00000 \\
$n=6$ & 1.00101 & 1.00017 & 1.00000 & 1.00000 & 1.00000 & 1.00000 & 1.00000 & 1.00000 & 1.00000 \\
$n=7$ & 1.00308 & 1.00037 & 1.00006 & 1.00000 & 1.00000 & 1.00000 & 1.00001 & 1.00004 & 1.00009 \\
$n=8$ & 1.00966 & 1.00263 & 1.00069 & 1.00015 & 1.00003 & 1.00003 & 1.00009 & 1.00014 & 1.00019 \\
$n=9$ & 1.01412 & 1.00413 & 1.00130 & 1.00023 & 1.00005 & 1.00004 & 1.00003 & 1.00005 & 1.00010 \\
$n=10$ & 1.01809 & 1.00552 & 1.00191 & 1.00061 & 1.00011 & 1.00005 & 1.00002 & 1.00003 & 1.00003 \\
$n=11$ & 1.01780 & 1.00523 & 1.00177 & 1.00065 & 1.00015 & 1.00006 & 1.00003 & 1.00002 & 1.00002 \\
$n=12$ & 1.01822 & 1.00463 & 1.00160 & 1.00059 & 1.00023 & 1.00011 & 1.00008 & 1.00004 & 1.00004 \\
$n=13$ & 1.02111 & 1.00538 & 1.00169 & 1.00066 & 1.00024 & 1.00012 & 1.00006 & 1.00003 & 1.00003 \\
$n=14$ & 1.01828 & 1.00463 & 1.00144 & 1.00054 & 1.00024 & 1.00016 & 1.00013 & 1.00006 & 1.00004 \\
$n=15$ & 1.01742 & 1.00511 & 1.00149 & 1.00060 & 1.00028 & 1.00013 & 1.00008 & 1.00007 & 1.00005 \\
\hline
\end{tabular}

Table 8. Mean of the ratio of expected posterior loss calculated by Algorithm 2 to the true minimized expectation, over 10,000 replicates from model 4, for various $K$ and $n$.

\begin{tabular}{llllllllll}
\hline \hline & $K=0.1$ & $K=0.2$ & $K=0.3$ & $K=0.4$ & $K=0.5$ & $K=0.6$ & $K=0.7$ & $K=0.8$ & $K=0.9$ \\
\hline$n=3$ & 1.00000 & 1.00000 & 1.00000 & 1.00000 & 1.00000 & 1.00000 & 1.00000 & 1.00000 & 1.00000 \\
$n=4$ & 1.00000 & 1.00000 & 1.00000 & 1.00000 & 1.00000 & 1.00000 & 1.00000 & 1.00000 & 1.00000 \\
$n=5$ & 1.00000 & 1.00000 & 1.00000 & 1.00000 & 1.00000 & 1.00000 & 1.00000 & 1.00000 & 1.00000 \\
$n=6$ & 1.00025 & 1.00000 & 1.00001 & 1.00000 & 1.00000 & 1.00000 & 1.00000 & 1.00000 & 1.00000 \\
$n=7$ & 1.00104 & 1.00013 & 1.00003 & 1.00001 & 1.00000 & 1.00000 & 1.00000 & 1.00000 & 1.00007 \\
$n=8$ & 1.00455 & 1.00119 & 1.00037 & 1.00007 & 1.00002 & 1.00003 & 1.00011 & 1.00020 & 1.00015 \\
$n=9$ & 1.00786 & 1.00243 & 1.00088 & 1.00023 & 1.00006 & 1.00004 & 1.00006 & 1.00011 & 1.00014 \\
$n=10$ & 1.00914 & 1.00312 & 1.00112 & 1.00037 & 1.00013 & 1.00005 & 1.00005 & 1.00013 & 1.00016 \\
$n=11$ & 1.01060 & 1.00332 & 1.00128 & 1.00048 & 1.00020 & 1.00008 & 1.00005 & 1.00005 & 1.00008 \\
$n=12$ & 1.00960 & 1.00325 & 1.00132 & 1.00049 & 1.00018 & 1.00011 & 1.00008 & 1.00005 & 1.00008 \\
$n=13$ & 1.00849 & 1.00257 & 1.00092 & 1.00046 & 1.00026 & 1.00013 & 1.00005 & 1.00003 & 1.00006 \\
$n=14$ & 1.00767 & 1.00268 & 1.00095 & 1.00049 & 1.00024 & 1.00011 & 1.00008 & 1.00003 & 1.00002 \\
$n=15$ & 1.00708 & 1.00201 & 1.00084 & 1.00035 & 1.00019 & 1.00013 & 1.00004 & 1.00004 & 1.00004 \\
\hline
\end{tabular}



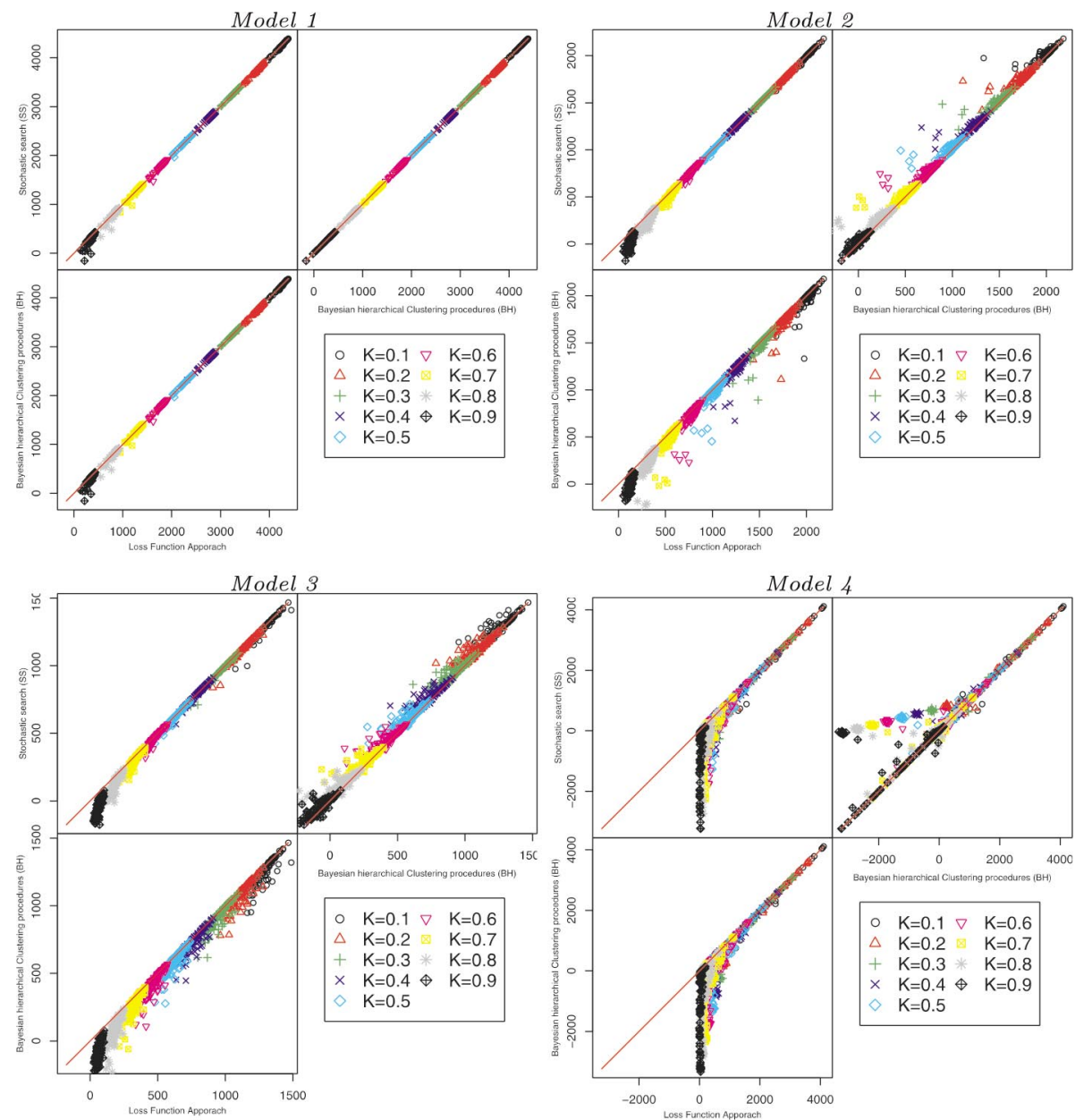

Figure 10. Pairwise comparisons of maximized log partition posterior probability, computed using the SS, BH, and Loss function approaches, under simulation models 1-4. In each of the four panels, the comparisons are: top left $=\mathrm{SS}$ versus loss function; top right $=\mathrm{SS}$ versus $\mathrm{BH}$; bottom left $=\mathrm{BH}$ versus loss function.

\subsubsection{Optimal Partition of Galaxy and Leukemia Datasets}

Now we consider the galaxy data. We calculate the $\rho$ matrix based on the last 10,000 partitions sampled. We run Algorithm 2 and the $\ell$ functions (defined by Equation (4.1)) for the partitions in $\mathcal{P}$ are plotted in Figure 12 (p. 552). Here we can see that we have the same best partition for all $K \in[0.1,0.9]$. Moreover, this partition is the same as that found by $\mathrm{SS}$ and $\mathrm{BH}$, and we conclude that for this dataset, the optimal partition for such values of $K$ coincides with the maximum a posteriori partition.

Finally we present the case of the leukemia data. Figure 13 (p. 552) shows $\ell(\mathcal{P}, K)$ over the interval $[0,1]$. We also plot the $\ell$ functions for the partitions produced by the SS and $\mathrm{BH}$ procedures. For this loss function, these approximate maximum a posteriori partitions are not optimal whatever the value of $K$, although the result from the $\mathrm{BH}$ procedure is close to optimal for $K \approx 0.08$. 

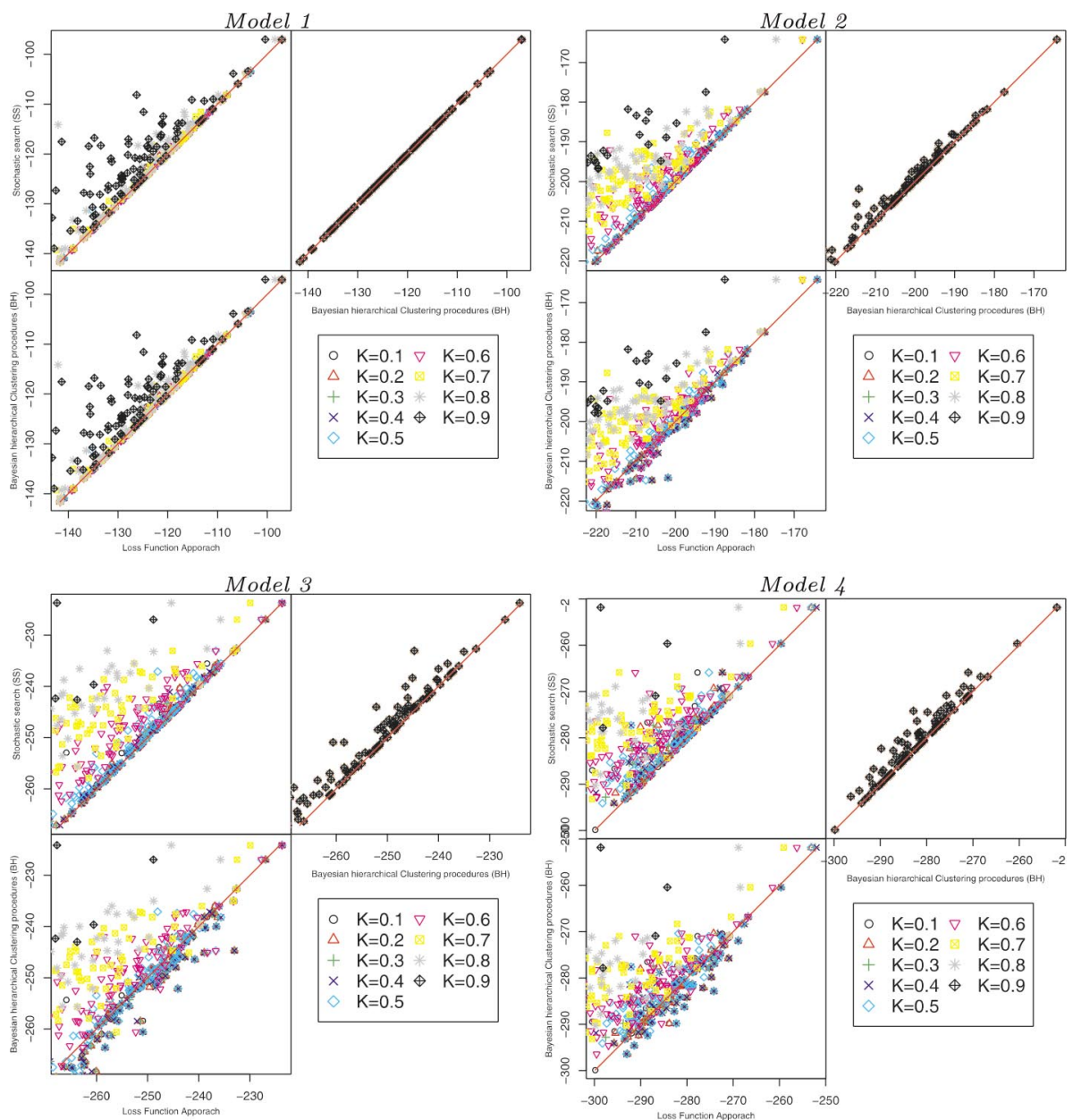

Figure 11. Pairwise comparisons of maximized objective function $\ell(\mathbf{p}, K)$, computed using the SS, BH, and Loss function approaches, under simulation models $1-4$. In each of the four panels, the comparisons are: top left $=\mathrm{SS}$ versus loss function; top right $=\mathrm{SS}$ versus $\mathrm{BH}$; bottom left $=\mathrm{BH}$ versus loss function.

We calculate $\log (\phi(\mathbf{p}))$ for the partitions generated by the algorithm for different $K \mathrm{~s}$, and these, together with the log posterior probabilities for the best partitions found by the SS and BH algorithms, are displayed in Table 9. It is interesting to note that in this example, for $K=0.1,0.2,0.3$, the highest posterior probability partitions are produced by our algorithm, which thus produces the best partitions under both the MAP and loss function criteria! Using the same computer resources as in the experiment in the last section, the computer program takes approximately 21 mins and 7,292 mins for the galaxy data and the leukemia data, respectively. 


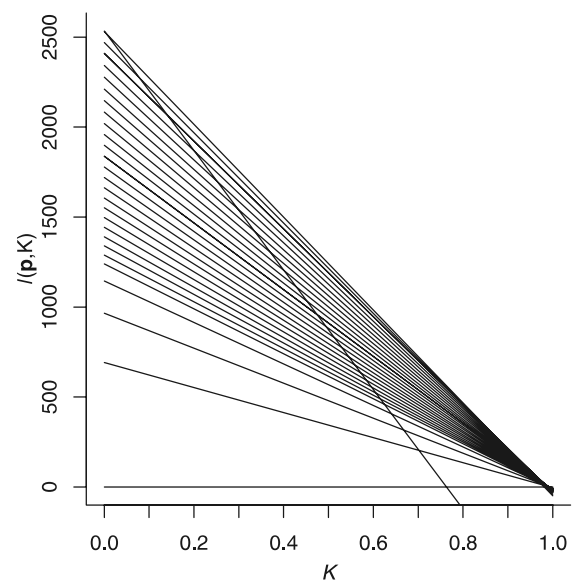

Figure 12. The objective function $\ell(\hat{\mathbf{p}}, K)$ versus $K$ for the partitions considered by Algorithm 2, for the galaxy data.

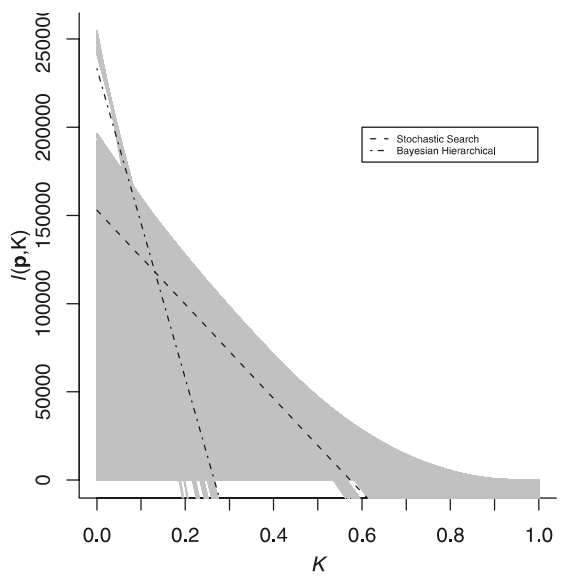

Figure 13. The objective function $\ell(\hat{\mathbf{p}}, K)$ versus $K$ for the partitions considered by Algorithm 2, for the leukemia data. 
Table 9. Log posterior probabilities for partitions computed by Algorithm 2, and by the SS and BH algorithms.

\begin{tabular}{cc}
\hline \hline & $\log (\phi(\mathbf{p}))$ \\
\hline$K=0.1$ & -262774.69 \\
0.2 & -262744.51 \\
0.3 & -262753.13 \\
0.4 & -263451.11 \\
0.5 & -265768.95 \\
0.6 & -270588.76 \\
0.7 & -274760.07 \\
0.8 & -279583.08 \\
0.9 & -286715.36 \\
& \\
SS & -263040.69 \\
BH & -263793.14 \\
\hline
\end{tabular}

\section{CONCLUSION}

Clustering is an important and challenging problem, with implications in many fields, including biology, engineering, and management. The highly complex structure of partition problems makes objective inference difficult, quite apart from the heavy computational burden entailed in any approach when the number of items to be clustered is large. A Bayesian formulation is attractive in principle, but can be particularly demanding computationally. However, the speed of modern processors is such that it is now practical to follow formal decision theoretic principles in partitioning a few thousand genes in Bayesian analysis of gene expression profiles. Computer time remains an issue, however. Further research should be conducted towards developing better algorithms to reduce the running time.

\section{A. APPENDIX}

\section{A.1 Proof of Equation (3.1)}

We first consider the ratio used to combine two clusters, say $C_{j_{1}}$ and $C_{j_{2}}$,

$$
\frac{m\left(\mathbf{y}_{C_{j_{1}} \cup C_{j_{2}}}\right)}{m\left(\mathbf{y}_{C_{j_{1}}}\right) m\left(\mathbf{y}_{C_{j_{2}}}\right)}=\exp \left\{-\left[\psi\left(\mathbf{y}_{C_{j_{1}} \cup C_{j_{2}}}\right)-\psi\left(\mathbf{y}_{C_{j_{1}}}\right)-\psi\left(\mathbf{y}_{C_{j_{2}}}\right)\right]\right\}
$$

and the exponent term is now

$$
\begin{aligned}
& \psi\left(\mathbf{y}_{C_{j_{1}} \cup C_{j_{2}}}\right)-\psi\left(\mathbf{y}_{C_{j_{1}}}\right)-\psi\left(\mathbf{y}_{C_{j_{2}}}\right) \\
& =\sum_{i \in C_{j_{1}} \cup C_{j_{2}}}^{n}\left(\mathbf{y}_{i}-\overline{\mathbf{y}}_{C_{j_{1}} \cup C_{j_{2}}}\right)^{\prime}\left(\mathbf{y}_{i}-\overline{\mathbf{y}}_{C_{j_{1}} \cup C_{j_{2}}}\right)
\end{aligned}
$$




$$
\begin{aligned}
& -\sum_{i \in C_{j_{1}}}^{n}\left(\mathbf{y}_{i}-\overline{\mathbf{y}}_{C_{j_{1}}}\right)^{\prime}\left(\mathbf{y}_{i}-\overline{\mathbf{y}}_{C_{j_{1}}}\right)-\sum_{i \in C_{j_{2}}}^{n}\left(\mathbf{y}_{i}-\overline{\mathbf{y}}_{C_{j_{2}}}\right)^{\prime}\left(\mathbf{y}_{i}-\overline{\mathbf{y}}_{C_{j_{2}}}\right) \\
& =\sum_{i \in C_{j_{1}}}^{n}\left(\mathbf{y}_{i}-\overline{\mathbf{y}}_{C_{j_{1}} \cup C_{j_{2}}}\right)^{\prime}\left(\mathbf{y}_{i}-\overline{\mathbf{y}}_{C_{j_{1}} \cup C_{j_{2}}}\right)-\sum_{i \in C_{j_{1}}}^{n}\left(\mathbf{y}_{i}-\overline{\mathbf{y}}_{C_{j_{1}}}\right)^{\prime}\left(\mathbf{y}_{i}-\overline{\mathbf{y}}_{C_{j_{1}}}\right) \\
& +\sum_{i \in C_{j_{2}}}^{n}\left(\mathbf{y}_{i}-\overline{\mathbf{y}}_{C_{j_{1}} \cup C_{j_{2}}}\right)^{\prime}\left(\mathbf{y}_{i}-\overline{\mathbf{y}}_{C_{j_{1}} \cup C_{j_{2}}}\right)-\sum_{i \in C_{j_{2}}}^{n}\left(\mathbf{y}_{i}-\overline{\mathbf{y}}_{C_{j_{2}}}\right)^{\prime}\left(\mathbf{y}_{i}-\overline{\mathbf{y}}_{C_{j_{2}}}\right) \\
& =e_{j_{1}}\left(\overline{\mathbf{y}}_{C_{j_{1}}}-\overline{\mathbf{y}}_{C_{j_{1}} \cup C_{j_{2}}}\right)^{\prime}\left(\overline{\mathbf{y}}_{C_{j_{1}}}-\overline{\mathbf{y}}_{C_{j_{1}} \cup C_{j_{2}}}\right)+e_{j_{2}}\left(\overline{\mathbf{y}}_{C_{j_{2}}}-\overline{\mathbf{y}}_{C_{j_{1}} \cup C_{j_{2}}}\right)^{\prime} \\
& \times\left(\overline{\mathbf{y}}_{C_{j_{2}}}-\overline{\mathbf{y}}_{C_{j_{1}} \cup C_{j_{2}}}\right) \\
& =e_{j_{1}} \overline{\mathbf{y}}_{C_{j_{1}}}^{\prime} \overline{\mathbf{y}}_{C_{j_{1}}}+e_{j_{2}} \overline{\mathbf{y}}_{C_{j_{2}}}^{\prime} \overline{\mathbf{y}}_{C_{j_{2}}}-\left(e_{j_{1}}+e_{j_{2}}\right) \overline{\mathbf{y}}_{C_{j_{1}} \cup C_{j_{2}}}^{\prime} \overline{\mathbf{y}}_{C_{j_{1}} \cup C_{j_{2}}} \\
& =\frac{e_{j_{1}} e_{j_{2}}}{e_{j_{1}}+e_{j_{2}}}\left(\overline{\mathbf{y}}_{C_{j_{1}}}-\overline{\mathbf{y}}_{C_{j_{2}}}\right)^{\prime}\left(\overline{\mathbf{y}}_{C_{j_{1}}}-\overline{\mathbf{y}}_{C_{j_{2}}}\right) \text {. }
\end{aligned}
$$

This equality will be useful:

$$
\begin{gathered}
\sum_{i \in C_{j_{1}} \cup C_{j_{2}}}^{n}\left(\mathbf{y}_{i}-\overline{\mathbf{y}}_{C_{j_{1}} \cup C_{j_{2}}}\right)^{\prime}\left(\mathbf{y}_{i}-\overline{\mathbf{y}}_{C_{j_{1}} \cup C_{j_{2}}}\right) \\
-\sum_{i \in C_{j_{1}}}^{n}\left(\mathbf{y}_{i}-\overline{\mathbf{y}}_{C_{j_{1}}}\right)^{\prime}\left(\mathbf{y}_{i}-\overline{\mathbf{y}}_{C_{j_{1}}}\right)-\sum_{i \in C_{j_{2}}}^{n}\left(\mathbf{y}_{i}-\overline{\mathbf{y}}_{C_{j_{2}}}\right)^{\prime}\left(\mathbf{y}_{i}-\overline{\mathbf{y}}_{C_{j_{2}}}\right) \\
=\frac{e_{j_{1}} e_{j_{2}}}{e_{j_{1}}+e_{j_{2}}}\left(\overline{\mathbf{y}}_{C_{j_{1}}}-\overline{\mathbf{y}}_{C_{j_{2}}}\right)^{\prime}\left(\overline{\mathbf{y}}_{C_{j_{1}}}-\overline{\mathbf{y}}_{C_{j_{2}}}\right) .
\end{gathered}
$$

\section{A.2 Proof of Equation (3.2)}

Similarly to the proof of (3.1), we need only consider the exponent term. In this case we first prove an equality

$$
\begin{aligned}
& \sum_{i_{1} \in C_{j_{1}}} \sum_{i_{2} \in C_{j_{2}}}\left(\mathbf{y}_{i_{1}}-\mathbf{y}_{i_{2}}\right)^{\prime}\left(\mathbf{y}_{i_{1}}-\mathbf{y}_{i_{2}}\right) \\
& =\sum_{i_{1} \in C_{j_{1}} \in C_{i_{2}}} \sum_{j_{2}}\left(\mathbf{y}_{i_{1}}-\overline{\mathbf{y}}_{C_{j_{1}}}+\overline{\mathbf{y}}_{C_{j_{1}}}-\overline{\mathbf{y}}_{C_{j_{2}}}+\overline{\mathbf{y}}_{C_{j_{2}}}-\mathbf{y}_{i_{2}}\right)^{\prime} \\
& \quad \times\left(\mathbf{y}_{i_{1}}-\overline{\mathbf{y}}_{C_{j_{1}}}+\overline{\mathbf{y}}_{C_{j_{1}}}-\overline{\mathbf{y}}_{C_{j_{2}}}+\overline{\mathbf{y}}_{C_{j_{2}}}-\mathbf{y}_{i_{2}}\right) \\
& =e_{j_{2}} \sum_{i_{1} \in C_{j_{1}}}\left(\mathbf{y}_{i_{1}}-\overline{\mathbf{y}}_{C_{j_{1}}}\right)^{\prime}\left(\mathbf{y}_{i_{1}}-\overline{\mathbf{y}}_{C_{j_{1}}}\right)+e_{j_{1}} e_{j_{2}}\left(\overline{\mathbf{y}}_{C_{j_{1}}}-\overline{\mathbf{y}}_{C_{j_{2}}}\right)^{\prime}\left(\overline{\mathbf{y}}_{C_{j_{1}}}-\overline{\mathbf{y}}_{C_{j_{2}}}\right) \\
& \quad+e_{j_{1}} \sum_{i_{2} \in C_{j_{2}}}\left(\overline{\mathbf{y}}_{C_{j_{2}}}-\mathbf{y}_{i_{2}}\right)^{\prime}\left(\overline{\mathbf{y}}_{C_{j_{2}}}-\mathbf{y}_{i_{2}}\right) .
\end{aligned}
$$

This is combined with the equality (A.1): 


$$
\begin{aligned}
& \psi\left(\mathbf{y}_{C_{j_{1}} \cup C_{j_{2}}}\right)-\psi\left(\mathbf{y}_{C_{j_{1}}}\right)-\psi\left(\mathbf{y}_{C_{j_{2}}}\right) \\
& =\left(e_{j_{1}}+e_{j_{2}}\right) \sum_{i \in C_{j_{1}} \cup C_{j_{2}}}\left(\mathbf{y}_{i}-\overline{\mathbf{y}}_{C_{j_{1}} \cup C_{j_{2}}}\right)^{\prime}\left(\mathbf{y}_{i}-\overline{\mathbf{y}}_{C_{j_{1}} \cup C_{j_{2}}}\right) \\
& -e_{j_{1}} \sum_{i \in C_{j_{1}}}\left(\mathbf{y}_{i}-\overline{\mathbf{y}}_{C_{j_{1}}}\right)^{\prime}\left(\mathbf{y}_{i}-\overline{\mathbf{y}}_{C_{j_{1}}}\right)-e_{j_{2}} \sum_{i \in C_{j_{2}}}\left(\mathbf{y}_{i}-\overline{\mathbf{y}}_{C_{j_{2}}}\right)^{\prime}\left(\mathbf{y}_{i}-\overline{\mathbf{y}}_{C_{j_{2}}}\right) \\
& =\sum_{i_{1} \in C_{j_{1}}} \sum_{i_{2} \in C_{j_{2}}}\left(\mathbf{y}_{i_{1}}-\mathbf{y}_{i_{2}}\right)^{\prime}\left(\mathbf{y}_{i_{1}}-\mathbf{y}_{i_{2}}\right) \\
& =\sum_{i_{1} \in C_{j_{1}}} \sum_{i_{2} \in C_{j_{2}}} d_{i_{1}, i_{2}} \text {. }
\end{aligned}
$$

\section{A.3 Proof of Equation (3.3)}

Again we consider only the exponent term. We first prove

$$
\begin{aligned}
& \psi\left(\mathbf{y}_{C_{j_{1}} \cup C_{j_{2}}}\right)-\psi\left(\mathbf{y}_{C_{j_{1}}}\right)-\psi\left(\mathbf{y}_{C_{j_{2}}}\right) \\
& =\sum_{i \in C_{j_{1}} \cup C_{j_{2}}}\left(\mathbf{y}_{i}-\left[\mathbf{x}_{1} \ldots \mathbf{x}_{S}\right]^{\prime} \hat{\boldsymbol{\beta}}_{C_{j_{1}} \cup C_{j_{2}}}\right)^{\prime}\left(\mathbf{y}_{i}-\left[\mathbf{x}_{1} \ldots \mathbf{x}_{S}\right]^{\prime} \hat{\boldsymbol{\beta}}_{C_{j_{1}} \cup C_{j_{2}}}\right) \\
& -\sum_{i \in C_{j_{1}}}\left(\mathbf{y}_{i}-\left[\mathbf{x}_{1} \ldots \mathbf{x}_{S}\right]^{\prime} \hat{\boldsymbol{\beta}}_{C_{j_{1}}}\right)^{\prime}\left(\mathbf{y}_{i}-\left[\mathbf{x}_{1} \ldots \mathbf{x}_{S}\right]^{\prime} \hat{\boldsymbol{\beta}}_{C_{j_{1}}}\right) \\
& -\sum_{i \in C_{j_{2}}}\left(\mathbf{y}_{i}-\left[\mathbf{x}_{1} \ldots \mathbf{x}_{S}\right]^{\prime} \hat{\boldsymbol{\beta}}_{C_{j_{2}}}\right)^{\prime}\left(\mathbf{y}_{i}-\left[\mathbf{x}_{1} \ldots \mathbf{x}_{S}\right]^{\prime} \hat{\boldsymbol{\beta}}_{C_{j_{2}}}\right) \\
& =\sum_{i \in C_{j_{1}}}\left(\left[\mathbf{x}_{1} \ldots \mathbf{x}_{S}\right]^{\prime} \hat{\boldsymbol{\beta}}_{C_{j_{1}}}-\left[\mathbf{x}_{1} \ldots \mathbf{x}_{S}\right]^{\prime} \hat{\boldsymbol{\beta}}_{C_{j_{1}} \cup C_{j_{2}}}\right)^{\prime} \\
& \times\left(\left[\mathbf{x}_{1} \cdot{ }_{3} . \mathbf{x}_{S}\right]^{\prime} \hat{\boldsymbol{\beta}}_{C_{j_{1}}}-\left[\mathbf{x}_{1} \ldots \mathbf{x}_{S}\right]^{\prime} \hat{\boldsymbol{\beta}}_{C_{j_{1}} \cup C_{j_{2}}}\right) \\
& +\sum_{i \in C_{j_{2}}}\left(\left[\mathbf{x}_{1} \ldots \mathbf{x}_{S}\right]^{\prime} \hat{\boldsymbol{\beta}}_{C_{j_{2}}}-\left[\mathbf{x}_{1} \ldots \mathbf{x}_{S}\right]^{\prime} \hat{\boldsymbol{\beta}}_{C_{j_{1}} \cup C_{j_{2}}}\right)^{\prime} \\
& \times\left(\left[\mathbf{x}_{1} \ldots \mathbf{x}_{S}\right]^{\prime} \hat{\boldsymbol{\beta}}_{C_{j_{2}}}-\left[\mathbf{x}_{1} \ldots \mathbf{x}_{S}\right]^{\prime} \hat{\boldsymbol{\beta}}_{C_{j_{1}} \cup C_{j_{2}}}\right) \\
& =\hat{\boldsymbol{\beta}}_{C_{j_{1}}}^{\prime} \mathbf{X}_{C_{j_{1}}}^{\prime} \mathbf{X}_{C_{j_{1}}} \hat{\boldsymbol{\beta}}_{C_{j_{1}}}+\hat{\boldsymbol{\beta}}_{C_{j_{2}}}^{\prime} \mathbf{X}_{C_{j_{2}}}^{\prime} \mathbf{X}_{C_{j_{2}}} \hat{\boldsymbol{\beta}}_{C_{j_{2}}}-\hat{\boldsymbol{\beta}}_{C_{j_{1}} \cup C_{j_{2}}}^{\prime} \\
& \times\left(\mathbf{X}_{C_{j_{1}}}^{\prime} \mathbf{X}_{C_{j_{1}}}+\mathbf{X}_{C_{j_{2}}}^{\prime} \mathbf{X}_{C_{j_{2}}}\right) \hat{\boldsymbol{\beta}}_{C_{j_{1}} \cup C_{j_{2}}} \\
& =\left(\hat{\boldsymbol{\beta}}_{C_{j_{1}}}-\hat{\boldsymbol{\beta}}_{C_{j_{2}}}\right)^{\prime}\left[\left(\mathbf{X}_{C_{j_{1}}}^{\prime} \mathbf{X}_{C_{j_{1}}}\right)^{-1}+\left(\mathbf{X}_{C_{j_{2}}}^{\prime} \mathbf{X}_{C_{j_{2}}}\right)^{-1}\right]^{-1}\left(\hat{\boldsymbol{\beta}}_{C_{j_{1}}}-\hat{\boldsymbol{\beta}}_{C_{j_{2}}}\right) \text {. }
\end{aligned}
$$

Finally, note that

$$
\mathbf{X}_{C_{j_{1}}}^{\prime} \mathbf{X}_{C_{j_{1}}}=\sum_{i \in C_{j_{1}}}\left[\mathbf{x}_{1} \ldots \mathbf{x}_{S}\right]\left[\mathbf{x}_{1} \ldots \mathbf{x}_{S}\right]^{\prime} \text { and } \mathbf{X}_{C_{j_{2}}}^{\prime} \mathbf{X}_{C_{j_{2}}}=\sum_{i \in C_{j_{2}}}\left[\mathbf{x}_{1} \ldots \mathbf{x}_{S}\right]\left[\mathbf{x}_{1} \ldots \mathbf{x}_{S}\right]^{\prime}
$$




\section{ACKNOWLEDGMENTS}

This work was carried out with the financial support of the BBSRC Exploiting Genomics initiative.

[Received June 2006. Revised January 2007.]

\section{REFERENCES}

Antoniak, C. E. (1974), "Mixtures of Dirichlet Processes with Applications to Bayesian Nonparametric Problems," The Annals of Statistics, 2, 1152-1174.

Banfield, J. D., and Raftery, A. E. (1993), “Model-Based Gaussian and Non-Gaussian Clustering,” Biometrics, 49, 803-821.

Bansal, N., Blum, A., and Chawla, S. (2004), "Correlation Clustering," Machine Learning, 56, 89-113.

Barry, D., and Hartigan, J. A. (1992), "Product Partition Models for Change Point Problems," The Annals of Statistics, 20, 260-279.

Basu, S., and Chib, S. (2003), "Marginal Likelihood and Bayes Factors for Dirichlet Process Mixture Models," Journal of the American Statistical Association, 98, 224-235

Berkelaar, M., et al. (2006), "IpSolve: Interface to Lp_solve v. 5.5 to Solve Linear/Integer Programs," R package version 5.5.3.

Binder, D. A. (1978), "Bayesian Cluster Analysis,” Biometrika, 65, 31-38.

- (1981), "Approximations to Bayesian Clustering Rules," Biometrika, 68, 275-285.

Blackwell, D., and MacQueen, J. B. (1973), "Ferguson Distributions via Pólya Urn Schemes," The Annals of Statistics, 1, 353-355.

Brunner, L. J., and Lo, A. Y. (1999), "Bayesian Classifications," Preprint, University of Toronto, Canada. Available online at http://www.erin.utoronto.ca/ jbrunner/papers/BayesClass.pdf

Dahl, D. B. (2006), "Model-Based Clustering for Expression Data via a Dirichlet Process Mixture Model," in Bayesian Inference for Gene Expression and Proteomics (chap. 10), eds. Kim-Anh Do, Peter Müller, and Marina Vannucci, Cambridge University Press.

de Finetti, B. (1930), "Funzione Caratteristica di un Fenomeno Aleatorio," Atti Reale Accademia Nazionale dei Lincei, Mem, 4, 86-133. (1974), Theory of Probability 1, New York: Wiley.

Diaconis, P., and Freedman, D. (1984), "Partial Exchangeability and Sufficiency," in Statistics: Applications and New Directions, eds. J. K. Ghosh and J. Roy, pp. 205-236.

(1987), “A Dozen de Finetti-style Results in Search of a Theory," Annales de l'Institut Henri Poincaré (B) Probabilités et Statistiques, 23, 397-423.

Dubey, A., Hwang, S., Rangel, C., Rasmussen, C. E., Ghahramani, Z., and Wild, D. L. (2004), “Clustering Protein Sequence and Structure Space with Infinite Gaussian Mixture Models," Pacific Symposium in Biocomputing, 9, 399-410.

Dudoit, S., Fridlyand, J., and Speed, T. P. (2002), "Comparison of Discrimination Methods for the Classification of Tumors using Gene Expression Data," Journal of the American Statistical Association, 97, 77-87.

Escobar, M. D., and West, M. (1995), "Bayesian Density Estimation and Inference using Mixtures," Journal of the American Statistical Association, 90, 577-588.

(1998), "Computing Monparametric Hierarchical Models," in Practical Nonparametric and Semiparametric Bayesian Statistics, eds. D. Dey, P. Müller, and D. Sinha, New York: Springer, pp. 1-22.

Ferguson, T. S. (1973), "A Bayesian Analysis of Some Nonparametric Problems," The Annals of Statistics, 1, 209-230.

Fraley, C., and Raftery, A. E. (2002), "Model-Based Clustering, Discriminant Analysis, and Density Estimation," Journal of the American Statistical Association, 97, 611-631.

Gentleman, R., et al. (2004), "Bioconductor: Open Software Development for Computational Biology and Bioinformatics," Genome Biology, 5, R80. http://genomebiology.com/2004/5/10/R80. 
Golub, T. R., Slonim, D. K., Tamayo, P., Huard, C., Gaasenbeek, M., Mesirov, J. P., Coller, H., Loh, M. L., Downing, J. R., Caligiuri, M. A., Bloomfield, C. D., and Lander, E. S. (1999), "Molecular Classification of Cancer: Class Discovery and Class Prediction by Gene Expression Monitoring," Science, 286, 531-537.

Gordon, A. D. (1999), Classification (2nd ed.), New York: Chapman \& Hall.

Green, P. J. (1995), "Reversible Jump Markov Chain Monte Carlo Computation and Bayesian Model Determination," Biometrika, 82, 711-732.

Green, P. J., and Richardson, S. (2001), "Modelling Heterogeneity With and Without the Dirichlet Process," Scandinavian Journal of Statistics, 28, 355-375.

Hartigan, J. A. (1990), "Partition Models," Communications in Statistics-Simulation and Computation, 19, 2745-2756.

Heard, N. A., Holmes, C. C., and Stephens, D. A. (2006), "A Quantitative Study of Gene Regulation Involved in the Immune Response of Anopheline Mosquitoes: An Application of Bayesian Hierarchical Clustering of Curves," Journal of the American Statistical Association, 101, 18-29.

Heller, K.A., and Ghahramani, Z. (2005), "Bayesian Hierarchical Clustering," Twenty-second International Conference on Machine Learning (ICML-2005). Available online at http://learning.eng.cam.ac.uk/zoubin/papers/ icml05heller.pdf.

Hewitt, E., and Savage, L. J. (1955), "Symmetric Measures on Cartesian Products," Transactions of the American Mathematical Society, 80, 470-501.

Hurn, M., Justel, A., and Robert, C. P. (2003), "Estimating Mixtures of Regressions," Journal of Computational and Graphical Statistics, 12, 55-79.

Ishwaran, H., and James, L. F. (2001), "Gibbs Sampling Methods for Stick-Breaking Priors," Journal of the American Statistical Association, 96, 161-173.

_ (2003a), "Generalized Weighted Chinese Restaurant Processes for Species Sampling Mixture Models," Statistica Sinica, 13 1211-1235.

_ (2003b), "Some Further Developments for Stick-Breaking Priors: Finite and Infinite Clustering and Classification," Sankhya Series A, 65, 577-592.

James, L. F. (2002), "Poisson Process Partition Calculus With Applications to Exchangeable Models and Bayesian Nonparametrics," available online at http://arXiv.org/abs/math/0205093.

(2005), "Bayesian Poisson Process Partition Calculus with an Application to Bayesian Levy Moving Averages," The Annals of Statistics, 33, 1771-1799.

James, L. F., Lijoi, A., and Prüenster, I. (2005), "Bayesian Inference for Classes of Normalized Random Measures," available online at http://arxiv.org/abs/math.ST/0503394.

Kingman, J. F. C. (1975), “Random Discrete Distributions,” Journal of the Royal Statistical Society, Series B, 37, $1-22$.

(1993), Poisson Processes, Cambridge, MA: Oxford University Press.

Kirkpatrick, S., Gelatt, C. D., and Vecchi, M. P., (1983), “Optimization by Simulated Annealing,” Science, 220, 671-680.

Lijoi, A., Mena, R. H., and Prünster, I. (2005), "Hierarchical Mixture Modeling with Normalized Inverse-Gaussian Priors," Journal of the American Statistical Association, 14, 1278-1291.

Lo, A. Y. (1984), “On a Class of Bayesian Nonparametric Estimates. I. Density Estimates," The Annals of Statistics, 12, 351-357.

(2005), "Weighted Chinese Restaurant Processes," COSMOS, 1, 59-63.

Lo, A. Y., Brunner, L. J., and Chan, A. T. (1996), "Weighted Chinese Restaurant Processes and Bayesian Mixture Models," Research Report, Hong Kong University of Science and Technology. Available online at http: //www.erin.utoronto.ca/ jbrunner/papers/wcr96.pdf

MacEachern, S. N. (1994), "Estimating Normal Means With a Conjugate Style Dirichlet Process Prior," Communications in Statistics-Simulation and Computation, 23, 727-741.

MacEachern, S. N., and Müller, P. (1998), "Estimating Mixture of Dirichlet Process Models," Journal of Computational and Graphical Statistics , 7, 223-238. 
(2000), "Efficient MCMC Schemes for Robust Model Extensions Using Encompassing Dirichlet Process Mixture Models," Robust Bayesian Analysis, 295-315.

Medvedovic, M., and Sivaganesan, S. (2002), "Bayesian Infinite Mixture Model Based Clustering of Gene Expression Profiles," Bioformatics, 18, 1194-1206.

Medvedovic, M., Yeung, K. Y., and Bumgarner, R. E. (2004), "Bayesian Mixture Model Based Clustering of Replicated Microarray Data," Bioformatics, 20, 1222-1232.

Neal, R. M. (2000), "Markov Chain Sampling Methods for Dirichlet Process Mixture Models," Journal of Computational and Graphical Statistics, 9, 249-265.

Nobile, A., and Fearnside, A. (2005), "Bayesian Finite Mixtures with an Unknown Number of Components: The Allocation Sampler," Technical Report 05-4, University of Glasgow. Available online at http://www.stats. gla.ac.uk/ agostino/mixalloc.pdf

Pitman, J. (1995), "Exchangeable and Partially Exchangeable Random Partitions," Probability Theory and Related Fields, 102, 145-158.

(1996), "Some Developments of the Blackwell-MacQueen Urn Scheme," in Statistics, Probability and Game Theory, Papers in honor of David Blackwell, IMS Lecture Note Series, eds. T. S. Ferguson, J. B. MacQueen, L. S. Shapley, pp. 245-267.

(2003), "Poisson Kingman Partitions," in Science and Statistics: A Festschrift for Terry Speed, IMS Lecture Note Series, eds. D. Goldstein, pp. 1-34. Available online at http://arxiv.org/abs/math/0210396.

Pitman, J., and Yor, M. (1997), "The Two-Parameter Poisson-Dirichlet Distribution Derived from a Stable Subordinator," The Annals of Probability, 25, 855-900.

Quintana F. A., and Iglesias P. L. (2003), "Bayesian Clustering and Product Partition Models," Journal of the Royal Statistical Society, Series B, 65, 557-574.

Ray, S., and Mallick, B. (2006), "Functional Clustering by Bayesian Wavelet Methods," Journal of the Royal Statistical Society, Series B, 68, 305-332.

Richardson, S., and Green, P. J. (1997), "On Bayesian Analysis of Mixtures With an Unknown Number of Components" (with discussion), Journal of the Royal Statistical Society, Series B, 59, 731-792.

Roeder, K. (1990), "Density Estimation with Confidence Sets Exemplified by Superclusters and Voids in the Galaxies," Journal of the American Statistical Association, 85, 617-624.

Rue, H. (1995), "New Loss Functions in Bayesian Imaging," Journal of the American Statistical Association, 90, 900-908.

Ward, J. H. (1963), "Hierarchical Grouping to Optimize an Objective Function," Journal of the American Statistical Association, 58, 236-244.

West, M., Müller, P., and Escobar, M. D. (1994), "Hierarchical Priors and Mixture Models, with Applications in Regression and Density Estimation," in A Tribute to D. V. Lindley, eds. A. F. M Smith and P. R. Freeman, New York: Wiley.

Yeung, K. Y., Fraley, C., Murua, A., Raftery, A. E., and Ruzzo, W. L. (2001), "Model-Based Clustering and Data Transformations for Gene Expression Data,” Bioinformatics, 17, 977-987. 